\title{
The mass-loss rates of Wolf-Rayet stars explained by optically thick radiation driven wind models
}

\author{
T. Nugis ${ }^{1}$ and H. J. G. L. M. Lamers ${ }^{2,3}$ \\ 1 Tartu Observatory, 61602 Tõravere, Estonia; e-mail: nugis@aai.ee \\ 2 Astronomical Institute, University of Utrecht, Princetonplein 5, 3584CC, Utrecht, The Netherlands; \\ e-mail: lamers@astro.uu.nl \\ 3 SRON Laboratory for Space Research, Utrecht, The Netherlands
}

Received 28 November 2001 / Accepted 9 April 2002

\begin{abstract}
Observed, clumping-corrected mass-loss rates of Galactic Wolf-Rayet (WR) stars are compared with predictions of the optically thick radiation driven wind models. We did not develop models for the whole wind, but we studied the conditions at the sonic point that would explain the observed high mass-loss rates of WR-stars. We find that optically thick wind models can explain the observed values of the mass-loss rates only if two conditions are satisfied:

(a) The sonic point (where $v_{\text {flow }}=v_{\text {sound }}$ ) lies deep in the wind where the temperature is either near $160000 \mathrm{~K}$, or in the range of 40000 to $70000 \mathrm{~K}$.

(b) The flux-mean opacity must increase outward from the sonic point.

With these conditions a simple approximate formula for the mass-loss rates of WR-stars can be derived.

The first condition implies that the sonic point is at an optical depth between about 3 and 30 . Such large optical depths require a slowly increasing velocity law in the supersonic region, with a velocity-law index of $\beta \simeq 5$ for WR-stars, compared to $\beta \simeq 1$ for O-stars. The OPAL-opacity tables for the chemical composition of the WR-stars show that the opacity indeed increases outward at the temperature range near $1.6 \times 10^{5} \mathrm{~K}$, and between about $4 \times 10^{7}$ and $7 \times 10^{4} \mathrm{~K}$, as required for the optically thick wind models. The opacity at the sonic points of the models is very similar to the OPAL-opacity at the sonic point temperature and density. The radius of the sonic point is about half as large as the inner boundaries of the "standard" models for early type WR-winds. Observational evidence, derived from line profile variations and from the light-curves of WR-stars in eclipsing binary systems, support the derived large values of $\beta$ and the small values of the sonic point radius. The models presented here show that the high mass-loss rates of WR-stars might be the result of optically thick radiation driven winds. The presence of two very distinct temperature regimes for the sonic point implies a bifurcation in the wind models of WR-stars.
\end{abstract}

Key words. stars: atmospheres - stars: mass-loss - stars: emission-lines - stars: evolution - stars: Wolf-Rayet

\section{Introduction}

Wolf-Rayet stars (WR-stars) are believed to be the evolved hot massive stars which almost have reached the end of their nuclear burning phase. Mass loss is a dominant feature of massive star evolution deeply influencing all stellar properties. The cause of the high mass loss from WR-stars has remained unveiled up to the present time.

It is often assumed that the winds of WR-stars are due to radiation pressure, i.e. the transfer of momentum from the radiation to the gas by means of absorption or scattering of photons, in analogy to the radiation driven winds of O-stars. This suggestion is based on the fact that both the luminosities of the WR-stars and the terminal wind velocities are in the same range as the luminosities

Send offprint requests to: T. Nugis, e-mail: nugis@aai.ee and wind velocities of the O-stars. However, the mass-loss rates of the WR-stars are about a factor ten higher than those of O-stars of the same luminosity. This implies that the transfer of momentum from the radiation to the gas must be about ten times more efficient than in the winds of O-stars. This high efficiency is needed both in the subsonic region deep in the wind, where the mass-loss rate is set, and in the supersonic region where the wind is accelerated to its high terminal velocity. The purpose of the present study is to find out whether optically thick wind models can provide sufficient radiative driving in the inner wind regions of WR-stars, i.e. whether the WR-winds are indeed radiatively driven.

Clumping-corrected mass-loss rates $(\dot{M})$ of WR-stars of different subtypes lie in the range of 0.2 to $10 \times$ $10^{-5} M_{\odot} \mathrm{yr}^{-1}$ and terminal velocities $\left(v_{\infty}\right)$ lie in the range 
700 to $6000 \mathrm{~km} \mathrm{~s}^{-1}$ (Nugis et al. 1998; Hamann et al. 2000; Nugis \& Lamers 2000). The luminosities of WR-stars are nearly of the same magnitude as O-stars but their masses are on the average 3 times lower (Maeder \& Meynet 1987; Nugis \& Lamers 2000). The momentum transfer efficiency $\eta$ of WR-stars, i.e. the ratio between the wind momentum loss and radiative momentum loss $\left(\eta=\dot{M} v_{\infty} /(L / c)\right)$, lies in the range of $1<\eta<20$. This high value of $\eta$ requires a very efficient momentum transfer with multiple scatterings (Owocki \& Gayley 1999). Lucy \& Abbott (1993) showed that if the actual $\eta$ is around 10 then the multiscattering in ionization-stratified WR-winds can provide the necessary driving force. According to Schmutz (1997) and Gayley et al. (1995) it is indeed possible to have sufficient radiative force for models with $\eta \approx 10$ to support the acceleration of the outer wind, but the mechanism that provides the driving in the inner wind regions, where $v<v_{\text {esc }}$ is still missing.

Owocki \& Gayley (1999) suggested that possibly a "two-stage" driving process might be needed, by which strong stellar pulsations actually initiate the mass loss, with radiative forces taking over to drive the extended acceleration and high terminal speeds. Cassinelli (1991) has suggested earlier that the high mass-loss rates of WR-stars may require a fast magnetic rotator model. In this paper we investigate whether there is indeed a need for an alternative mechanism to initiate the winds of WR-stars, or whether radiation pressure in the optically thick transonic layers is sufficiently efficient to get the wind started.

Line-driven wind models are quite successful in explaining the observed properties of mass loss from OBstars. Unfortunately, a straightforward application of these models to WR-stars is not justified. The main acceleration in the WR-winds takes place at large values of frequency-averaged optical depths, i.e. below the photosphere, where radiation forces due to true continuum absorption are important. In the line driven wind models of O-stars these can be ignored. The first attempts to apply "optically thick wind" to WR-stars have been made by Kato \& Iben (1992) and by Pistinner \& Eichler (1995). An optically thick wind is usually regarded as a steady state wind in which the acceleration of matter is due to continuum absorption, occuring below the photosphere (Kato \& Iben 1992). In the present paper we define an "optically thick wind" as a steady state radiatively driven wind with the sonic point located at large optical depth $(\tau \gg 1)$. In our definition the opacity below the photosphere is not necessarily dominated by continuum absorption. All opacity sources must be taken into account: line absorption, continuum absorption and electron scattering. Recent recalculations of Rosseland mean opacities (Iglesias \& Rogers 1993, 1996) have revealed increased opacities in the outer stellar layers as compared to older calculations and this is basically due to a more correct inclusion of metal bound-bound transitions. The full self-consistent solution of optically thick winds requires the computation of a model for the whole star which takes into account both the nuclear burning core and the radiatively expanding envelope. This is a complicated task that requires the knowledge of accurate opacities and physics in the dynamical, i.e. expanding envelope. At present, this knowledge is not available. Therefore we undertake a simpler task: we investigate whether optically thick wind models of WR-stars can explain the observed mass-loss rates and we derive the specific properties of $W R$-stars at low expansion velocities below the sonic point. To achieve this, we take the following steps:

(a) We adopt the relations between the luminosity and the mass of the WR-stars as predicted by the evolutionary calculations. This is justified because it is well known that the basic properties of stellar cores of WR-stars are not depending on the ways and processes how the mass is removed from outer layers (Maeder \& Meynet 1987; Langer 1989; Maeder 1991).

(b) We derive the equations that describe the structure of the optically thick part of the wind. These are the coupled equations of mass continuity, radiative transfer, energy conservation and momentum conservation. The temperature structure follows from the energy equation and the velocity structure follows from the momentum equation. The opacities due to lines and continuum play a crucial role in determining both the temperature structure, via the heating and cooling terms and the optical depth, and the velocity structure because the radiative force depends on the flux-mean opacity.

(c) Since we do not know the opacities with sufficient accuracy to construct a full optically thick wind model, we will express the temperature and velocity structure as a function of the optical depth. The optical depth depends on the structure of the atmosphere in both the subsonic and the supersonic region. For the supersonic region we will use the empirically determined velocity and density structure for the calculation of the optical depth.

(d) The mass-loss rate of any stellar wind model is set by the condition that the solution of the momentum equation should smoothly pass through its critical point, which is usually the sonic point (e.g. Lamers \& Cassinelli 1999). We investigate the conditions at the sonic point in the optically thick wind to see if they can explain the observed high mass-loss rates of the WR-stars.

(e) We will show that the observed mass-loss rates can be explained by radiation pressure in an optically thick wind, but only if the velocity law in the supersonic part of the wind is much softer (slower acceleration) than generally adopted. This requirement comes from the fact that the optical depth at the sonic point must already be very much larger than unity.

(f) We then compare the opacities that are needed to explain the high mass-loss rates of the WR-stars by means of optically thick radiation driven winds with new OPAL opacities.

The structure of the paper is as follows. In Sect. 2 we describe the physical processes and the mathematical equations of the optically thick radiation driven wind models. In Sect. 3 we describe the method that was used to calculate the optically thick wind models and in Sect. 4 
we give some simple estimates based on general momentum and energy considerations of optically thick winds. In Sect. 5 we present the results of a few series of test models for the optically thick wind of the star WR139 (WN5). We will draw some important conclusions about the properties and the conditions of optically thick wind models. In Sects. 6 and 7 we apply the optically thick wind models to explain the observed mass-loss rates of a set of characteristic WN and WC stars. In Sect. 6 we first discuss the characteristic properties of these stars, such as the adopted radius of the sonic point. The models for these stars are presented in Sect. 7. In Sect. 8 we make new models with the opacity gradient at the sonic point derived from the OPAL-opacities for the abundances of WR-stars and we discuss the existence of a bifurcation in the wind models of WR-stars. The results are discussed in Sect. 9 and the conclusions are in Sect. 10.

\section{Optically thick wind models}

In this section we will derive the equations that describe the flow of the radiation and gas in the optically thick part of the wind. The wind has to be accelerated from subsonic velocities deep down in the atmosphere to supersonic velocities in the wind. This implies that the wind must pass smoothly through the sonic point where the flowspeed is equal to the local sound speed. We first describe the general equations for radiation driven winds. We then apply these to the sonic point. The requirement of a smooth transonic flow sets specific conditions to the values and gradients of several parameters, including the mass-loss rate. We will derive these conditions.

\subsection{General equations for radiation driven winds}

We assume that the winds of WR-stars can be described by a steady, spherically-symmetric flow. The fluid flow equations for radiatively driven winds are:

$\frac{\mathrm{d}}{\mathrm{d} r}\left(\rho v r^{2}\right)=0$

$v \frac{\mathrm{d} v}{\mathrm{~d} r}+\frac{1}{\rho} \frac{\mathrm{d} P_{\mathrm{g}}}{\mathrm{d} r}=-\frac{G M}{r^{2}}+f_{\mathrm{rad}}$,

$v \frac{\mathrm{d} U_{\mathrm{g}}}{\mathrm{d} r}+P_{\mathrm{g}} v \frac{\mathrm{d}}{\mathrm{d} r}\left(\frac{1}{\rho}\right)=4 \pi \int_{0}^{\infty} 4 \pi \chi_{\nu}\left(J_{\nu}-S_{\nu}\right) \mathrm{d} \nu$.

These equations express conservation of respectively mass, momentum and energy of the gas. The symbols are the mass density $\rho$, the gas pressure $P_{\mathrm{g}}$, the velocity $v$, the (angle-) mean intensity of radiation $J_{\nu}$, the mass extinction (absortion + scattering) coefficient $\chi_{\nu}$, the radiative source function $S_{\nu}$, the internal energy $U_{\mathrm{g}}$ of the gas per unit mass, the stellar mass $M$ and the force $f_{\text {rad }}$ produced by radiation pressure. The mass-loss rate of the star is

$\dot{M}=4 \pi \rho v r^{2}$.
The gas pressure $P_{\mathrm{g}}$ depends on temperature $T$ and density $\rho$ as

$P_{\mathrm{g}}=\frac{(\gamma+1)}{\mu m_{\mathrm{u}}} \rho k T$,

where $\gamma$ is the mean number of free electrons per atom, $\mu m_{\mathrm{u}}$ is the mean atomic weight and $m_{\mathrm{u}}$ is the atomic mass unit. The internal energy $U_{\mathrm{g}}$ is related to $P_{\mathrm{g}}$ by the rule

$U_{\mathrm{g}}=\frac{3}{2} \frac{P_{\mathrm{g}}}{\rho}$.

The isothermal sound speed is given by

$v_{\mathrm{s}}^{2}=\mathrm{d} P_{\mathrm{g}} / \mathrm{d} \rho=(\gamma+1) k T /\left(\mu m_{\mathrm{u}}\right)$.

\subsubsection{The momentum equation}

Using the formulae (1), (5) and (7) we can transform the momentum Eq. (2) into the form

$\left(v-\frac{v_{\mathrm{s}}^{2}}{v}\right) \frac{\mathrm{d} v}{\mathrm{~d} r}=\frac{2 v_{\mathrm{s}}^{2}}{r}-\frac{G M}{r^{2}}-\frac{v_{\mathrm{s}}^{2}}{T} \frac{\mathrm{d} T}{\mathrm{~d} r}+f_{\mathrm{rad}}$.

The radiation pressure force is

$f_{\mathrm{rad}}=\frac{4 \pi}{c} \int_{0}^{\infty} \chi_{\nu} H_{\nu} \mathrm{d} \nu=\frac{\chi L(r)}{4 \pi r^{2} c}$

where $H_{\nu}$ is the first moment of the radiation intensity (the Eddington flux), $L(r)$ is the radiative luminosity in the frame comoving with the wind and $\chi$ is the flux-mean opacity (the flux-mean extinction (absorption + scattering) coefficient per unit mass).

\subsubsection{The energy equation}

The integrated overall energy conservation equation for both the gas and the radiation is

$L(r)+L_{\mathrm{adv}}(r)+\dot{M}\left\{\frac{v^{2}}{2}-\frac{G M}{r}+\frac{P_{\mathrm{g}}}{\rho}+U_{\mathrm{g}}\right\}=\dot{E}$,

where $\dot{E}$ is the energy-loss constant (the total energy transported out per unit time across any spherical surface). The first term is the luminosity $L(r)$ in the comoving frame. The second term is the radiative energy that is carried out (advected) by the gas flow in the comoving frame. Cassinelli \& Castor (1973) have shown that the advective luminosity $L_{\mathrm{adv}}$ is

$L_{\mathrm{adv}}=16 \pi^{2} r^{2} \frac{v}{c}(J+K)$.

This term is independent of $\rho$ because the radiation density is independent of $\rho$. At large distance from the star the moments of the radiation approach $J=K=H=$ $L(r=\infty) /\left(16 \pi^{2} r^{2}\right)$ and so

$L_{\mathrm{adv}}(\infty)=\frac{2 v_{\infty} L(\infty)}{c}$

The sum of $L(r)$ and $L_{\mathrm{adv}}$ is the luminosity in the stationary frame (see Cassinelli \& Castor 1973; 
Pistinner \& Eichler 1995). The third term in Eq. (10) is the total gas energy (kinetic, thermal and potential) that is advected by the flow.

The energy-loss constant $\dot{E}$ can be found from general considerations. The energy rate $L_{\text {core }}$ generated by the nuclear fusion must be equal to the luminosity at infinity plus the energy lost by accelerating the wind and lifting it out of the potential well (cf. Heger \& Langer 1996):

$L_{\text {core }} \simeq L(\infty)\left(1+\frac{2 v_{\infty}}{c}\right)+\dot{M}\left(\frac{v_{\infty}^{2}}{2}+\frac{G M}{R_{\mathrm{hc}}}\right)$,

where $R_{\mathrm{hc}}$ is the radius of the hydrostatic core. We see from Eqs. (10) and (12) that

$L(\infty)\left(1+\frac{2 v_{\infty}}{c}\right)+\dot{M} \frac{v_{\infty}^{2}}{2} \approx \dot{E}$.

From these two equations we obtain an expression for the energy loss constant

$\dot{E}=L_{\text {core }}-\dot{M} \frac{G M}{R_{\mathrm{hc}}}$.

\subsection{The optically thick part of the wind}

The momentum Eq. (8) and the energy Eq. (10) together describe the radiation driven wind. The solution of these equations requires knowledge about the opacity and the temperature structure. For the optically thick part of the wind we can make some simplifying approximations, because the transfer of radiation can be described by diffusion. In the case of radiative diffusion the temperature gradient is

$\frac{\mathrm{d} T}{\mathrm{~d} r}=\frac{-3 \chi_{\mathrm{R}} \rho L(r)}{64 \pi r^{2} \sigma T^{3}}$,

and the radiation-pressure force is

$f_{\mathrm{rad}}=\frac{\chi_{\mathrm{R}} L(r)}{4 \pi r^{2} c}$

where $\chi_{\mathrm{R}}$ is the Rosseland mean opacity. In the optically thick part of the wind the mean intensity of the radiation is $J=\sigma T^{4} / \pi$ and $K=J / 3$ (Cassinelli \& Castor 1973) and so the advective luminosity (Eq. (11)) is

$L_{\mathrm{adv}}=16 \pi^{2} r^{2} \frac{v}{c} \frac{4 \sigma T^{4}}{3 \pi}$.

Defining the constants $c_{1}=64 \pi \sigma /(3 c)=1.267 \times 10^{-13}$ and $a_{1}=k(\gamma+1) /\left(\mu m_{\mathrm{u}}\right)=8.314 \times 10^{7}(\gamma+1) / \mu$ in cgsunits, where $\gamma$ is the mean number of free electrons per ion, we can write the energy equation in the optically thick part of the wind as

$L(r)=\dot{E}-c_{1} r^{2} v T^{4}-\dot{M}\left(\frac{v^{2}}{2}-\frac{G M}{r}+\frac{5 a_{1} T}{2}\right)$.

This is the form of the energy equation that we will use to find the radiative luminosity $L(r)$ in the comoving frame that is needed for the calculation of the radiative force $f_{\text {rad }}$.
The diffusion approximation becomes valid at large optical depths (i.e., many photon mean-free-paths below the surface) where $T \gg T_{\text {eff }}$ (Mihalas 1978, p. 49). For an estimate, let us adopt that if the photon mean-free-path is more than 10 times smaller than the sonic point radius then the diffusion approximation is valid. The photon mean-free-path $\left(\overline{l_{\nu}}\right)$ is defined as $1 /(\rho \chi)$. Using the sonic point parameters from the paper (see Table 7 ), we find that in the case of the WN6 star WR136 $\overline{l_{\nu}} \approx 2.38 \times 10^{9} \mathrm{~cm}$ $\left(\overline{l_{\nu}} / R_{\mathrm{S}} \approx 0.0074\right)$ and in the case of the WN5 star WR 139 $\overline{l_{\nu}} \approx 1.82 \times 10^{9} \mathrm{~cm}\left(\overline{l_{\nu}} / R_{\mathrm{s}} \approx 0.013\right)$. Thus, the diffusion approximation is valid for the regions near the sonic points of WR-star winds. Note that this is not the case for the winds of O-stars, which generally have $\overline{l_{\nu}} / R_{\mathrm{s}}>0.1$.

\subsection{The conditions at the sonic point}

The momentum Eq. (8) has the characteristic form of an equation with a critical point. The left hand side shows that when $v=v_{\mathrm{s}}$, the velocity gradient $\mathrm{d} v / \mathrm{d} r$ becomes infinite (positive or negative), unless the right hand side is zero at this sonic point. So the right hand side must vanish exactly at the critical point. The smooth passage of the flow through the sonic point also requires that the derivatives of the temperature and velocity are equal on both sides of the sonic point. These conditions are expressed by the de l'Hopital rule which describes the relations for the radial velocity gradients at the critical point (see e.g. Lamers \& Cassinelli 1999, p. 421).

The momentum Eq. (8) can be written as

$f_{1}=f_{2} \frac{\mathrm{d} v}{\mathrm{~d} r}$

with

$f_{1}=\frac{2 a_{1} T}{r}-\frac{G M}{r^{2}}-a_{1} \frac{\mathrm{d} T}{\mathrm{~d} r}+\frac{\chi L(r)}{4 \pi c r^{2}}$

and

$f_{2}=v-\frac{a_{1} T}{v}$.

The conditions at the sonic point, $R_{\mathbf{s}}$, where the flow velocity is

$v_{\mathrm{s}} \equiv v\left(R_{\mathrm{s}}\right)=\sqrt{a_{1} T_{\mathrm{s}}}$

with $T_{\mathrm{s}} \equiv T\left(R_{\mathrm{s}}\right)$ are

$f_{1}\left(R_{\mathrm{S}}\right)=0 \quad$ and $\quad f_{2}\left(R_{\mathbf{s}}\right)=0$.

The first condition implies that

$\chi_{\mathrm{s}} L_{\mathrm{s}}=4 \pi c R_{\mathrm{s}}^{2}\left(-\frac{2 a_{1} T_{\mathrm{s}}}{R_{\mathrm{s}}}+\frac{G M}{R_{\mathrm{s}}^{2}}+a_{1}\left(\frac{\mathrm{d} T}{\mathrm{~d} r}\right)_{\mathrm{s}}\right)$,

where the subscript $\mathrm{s}$ denotes the values at the sonic point. The velocity gradient at the sonic point is given by de l'Hopital's rule

$\left(\frac{\mathrm{d} v}{\mathrm{~d} r}\right)_{\mathrm{s}}=\left(\frac{\mathrm{d} f_{1}}{\mathrm{~d} r}\right)_{\mathrm{s}} /\left(\frac{\mathrm{d} f_{2}}{\mathrm{~d} r}\right)_{\mathrm{s}}$. 
Since $f_{2}$ increases from negative below the sonic point to positive above the sonic point, the gradient $\mathrm{d} f_{1} / \mathrm{d} r$ should be positive at the sonic point. In physical terms this means that either energy or momentum must be added to the wind as it passes through the sonic point (cf. Lamers \& Cassinelli 1999, p. 100).

The requirement that the velocity gradient should be continuous through the sonic point (i.e. the regularity condition) implies that

$$
\left(\frac{\mathrm{d}^{2} v}{\mathrm{~d} r^{2}}\right)_{\mathrm{s}}=\frac{\left(\frac{\mathrm{d}^{2} f_{1}}{\mathrm{~d} r^{2}}-\frac{\mathrm{d} v}{\mathrm{~d} r} \frac{\mathrm{d}^{2} f_{2}}{\mathrm{~d} r^{2}}-\frac{\mathrm{d} f_{2}}{\mathrm{~d} r} \frac{\mathrm{d}^{2} v}{\mathrm{~d} r^{2}}\right)_{\mathrm{S}}}{\left(\frac{\mathrm{d} f_{2}}{\mathrm{~d} r}\right)_{\mathrm{s}}}
$$

with the derivatives $\mathrm{d} f_{1} / \mathrm{d} r, \mathrm{~d} f_{2} / \mathrm{d} r, \mathrm{~d}^{2} f_{1} / \mathrm{d} r^{2}$ and $\mathrm{d}^{2} f_{2} / \mathrm{d} r^{2}$ following from the definitions of $f_{1}$ and $f_{2}$.

We point out that the CAK-type line forces (actually the line force amplification due to Doppler shifts - Castor et al. 1975) can be neglected near the sonic point of WRstar winds. The CAK-type line force is usually expressed in relation to the radiation force by electron scattering as follows

$f_{\mathrm{L}}=\frac{\sigma_{\mathrm{e}}^{\mathrm{ref}} M(t) L}{4 \pi c r^{2}}$

where $\sigma_{\mathrm{e}}^{\text {ref }}$ is the reference value for the electron scattering opacity $\left(\sigma_{\mathrm{e}}^{\mathrm{ref}}=0.325 \mathrm{~cm}^{2} \mathrm{~g}^{-1}\right.$, Lamers \& Cassinelli 1999 , p. 217). The force multiplier can be expressed as

$$
M(t)=k t^{-\alpha} C,
$$

where $k$ and $\alpha$ are the force multiplier parameters, $C$ is a correction term which is very close to unity and $t$ is the dimensionless optical depth parameter defined as

$t=\frac{\sigma_{\mathrm{e}}^{\mathrm{ref}} v_{\mathrm{th}} \rho}{\mathrm{d} v / \mathrm{d} r}$

where $v_{\text {th }}$ is the mean thermal velocity of protons. Our optically thick wind models show that $\mathrm{d} v / \mathrm{d} r \approx v_{\mathrm{s}} / R_{\mathrm{s}}$ near the sonic point. The value of $t$ at the sonic point can be found from the relationship

$$
t=\frac{\sigma_{\mathrm{e}}^{\mathrm{ref}} \sqrt{(2 \mu /(\gamma+1))} \dot{M}}{4 \pi v_{\mathrm{s}} R_{\mathrm{s}}} .
$$

This shows that near the sonic point of the WN6 star WR136 $t \approx 140\left(R_{\mathrm{s}} \approx 4.6 R_{\odot}\right)$ and near the sonic point of WN5 star WR139t $\approx 52\left(R_{\mathrm{s}} \approx 2.0 R_{\odot}\right)$. With such large values of $t$ and with the expected values of $\alpha$ $(\approx 0.5)$ and $k(\approx 0.6-0.7)$, the force multiplier is indeed very small, $M(t)<0.05$ to 0.08 . This implies that the CAK-type line force due to Doppler shifts is negligible compared to electron scattering and other radiation forces near the sonic points of WR-stars. One might counterargue that the velocity gradient near the sonic point might be of order $\mathrm{d} v / \mathrm{d} r \approx v_{\infty} / R_{\mathrm{s}}$, as in optically thin (in the continuum) CAK-type O-star wind models, rather than $\mathrm{d} v / \mathrm{d} r \approx v_{\mathrm{s}} / R_{\mathrm{s}}$. If that were the case, the values of $t$ near the sonic points of WR-stars would be smaller than given above $(t \approx 3$ for WR136 and $t \approx 1$ for WR139), but the force multiplier is still very small $0.3<M(t)<0.6$ compared to $t \approx 10^{-2}$ and $M(t)>10^{1}$ for O-stars. In this estimate we neglected continuum absorption within the Sobolev length. This implies that the true CAK-forces near the sonic points of WR-star winds are even smaller than estimated above. So, we can safely conclude that near the sonic points of WR-star winds the CAK-type forces are not important, but they become the dominate driving force starting from some distance above the sonic point. The influence of these forces is not ignored in our models - they are taken into account indirectly by using the momentum equation in the supersonic part of the wind by assuming that the winds are radiatively driven.

\subsection{The temperature structure}

The conditions at the sonic point, described above, contain the derivatives of the temperature $T(r)$, which depends on the velocity $v(r)$ structure. In this section we describe these two functions. From the analytical solution of the spherically extended atmosphere in radiative equilibrium in the generalized Eddington approximation derived by Lucy (1971) we find that

$T^{4}(r) \approx \frac{3}{4} T_{\mathrm{eff}}^{4}\left(R_{\mathrm{s}}\right)\left(\tau^{\prime}+\frac{4}{3} W(r)\right)$.

In this expression $T_{\text {eff }}\left(R_{\mathrm{S}}\right) \equiv\left(L /\left(4 \pi \sigma R_{\mathrm{S}}^{2}\right)\right)^{0.25}$ where $R_{\mathrm{S}}$ is the radius of the sonic point of the wind, $W(r)$ is the coefficient of geometrical dilution, and the effective optical depth $\tau^{\prime}$ is defined by

$\tau^{\prime}=\int_{r}^{\infty} \chi \rho \frac{R_{\mathrm{s}}^{2}}{r^{2}} \mathrm{~d} r$,

where $\chi$ is the flux-mean extinction coefficient. We point out that Heger \& Langer (1996) and Lucy \& Abbott (1993) used a slightly different version of the original Lucy (1971) formula. They used the photospheric radius $\left(R_{\mathrm{ph}}\right.$ where $\left.\tau^{\prime}\left(R_{\mathrm{ph}}\right)=2 / 3\right)$ as a reference level, whereas below the photospheric radius the geometrical dilution was neglected. Test computations have shown that our results (described below) would have been qualitatively the same if we had adopted this same reference level $R_{\mathrm{ph}}$ instead of the value of $R_{\mathrm{s}}$ that we adopted. The important argument against the use of $R_{\mathrm{ph}}$ as reference level is the large difference between $R_{\mathrm{ph}}$ and $R_{\mathrm{s}}$ for WR-stars. Therefore the effects of geometrical dilution should not be neglected in the region below $R_{\mathrm{ph}}$, where $J / K$ is not constant. Our approach is almost in line with the arguments of the optically thick wind modeling study of the $1.4 M_{\odot}$ stars driven by super-Eddington luminosities by Quinn \& Paczyński (1985). They stated (p. 635 in their paper) that "Models of radiatively driven winds with a critical point at a large optical depth have very diffuse photospheres with the density scale height approximately equal to the local radius. Therefore, the variation of radiation energy density with radius is due not only to diffusion through opaque matter, but also to geometrical dilution". 
We adopt the temperature structure of a spherically symmetric stationary wind in radiative equilibrium, given by Eq. (32) with the effective optical depth given by Eq. (33). This approximation is not valid at distances larger than $r \sim R(\tau \approx 2 / 3)$, because it assumes that $J \approx B$. For example Eq. (32) predicts that at large distance from the star, where $\tau^{\prime}$ becomes very small, the temperature will go to zero. In reality the observations and empirical wind models show that the temperatures of WR-winds approach a constant finite value of order $10^{4} \mathrm{~K}$ at distances of a few tens of stellar radii. This is due to the radiative heating by photoionization. Nugis et al. (1998) have determined the asymptotic temperatures $T_{\text {asymp }}$ of WR-winds as a function of WR-subtypes. We adopt these values. So the temperature structure of our models is described by Eq. (32) if $T(r) \geq T_{\text {asymp }}$ and $T(r)=T_{\text {asymp }}$ where Eq. (32) would predict a smaller value. We should point out that the choice of $T_{\text {asymp }}$ plays only a minor role in our modeling, as most of the important physical effects occur in the optically thick part of the wind.

The temperature at the sonic point is

$T_{\mathrm{s}}^{4}=\frac{3}{4} T_{\text {eff }}^{4}\left(R_{\mathrm{s}}\right)\left(\tau_{\mathrm{s}}^{\prime}+2 / 3\right)$,

with an effective optical depth $\tau_{\mathrm{s}}^{\prime}$ at the sonic point

$\tau_{\mathrm{s}}^{\prime}=\int_{R_{\mathrm{s}}}^{\infty} \chi \rho \frac{R_{\mathrm{s}}^{2}}{r^{2}} \mathrm{~d} r$

Notice that $T_{\mathrm{s}}$ depends on the effective optical depth at the sonic point and hence on the run of $\chi \rho$ at all layers above $R_{\mathrm{s}}$. Therefore we can only find the solution of the momentum and energy equations at the sonic point if we know the functions $\chi(r)$ and $\rho(r)$ at $r>R_{\mathrm{s}}$.

For determining the distribution of the flux-mean opacity in the wind, we use the approach of Lucy \& Abbott (1993) who derived $\chi(r)$ from the demand of the momentum conservation in the wind for an adopted velocity law. This requirement follows from Eqs. (8) and (16):

$\chi=\frac{4 \pi c r^{2}}{L(r)}\left\{\left(v-\frac{a_{1} T}{v}\right) \frac{\mathrm{d} v}{\mathrm{~d} r}-\frac{2 a_{1} T}{r}+\frac{G M}{r^{2}}+a_{1} \frac{\mathrm{d} T}{\mathrm{~d} r}\right\}$.

So for a given $v(r), T(r)$ and $L(r)$ the function $\chi(r)$ is known. Note that $\chi$ and $v$ are related so that $\chi$ increases with the increase of $v$. The term consisting of $\mathrm{d} T / \mathrm{d} r$ is very small and is found by using the diffusion approximation for $r \leq 1.01 R_{\mathrm{s}}$ and by differentiating the temperature formula for $r>1.01 R_{\mathrm{s}}$.

The value of $L(r)$ is very close to $L_{\text {core }}$ throughout the optically thick and optically thin part of the wind. This is because the other terms in the energy Eq. (19), i.e. $L_{\mathrm{adv}}$ and the flow of potential, kinetic energy and enthalpy, are all very small compared to $L_{\text {core }}$ for WR-stars. So in principle we could have used $L(r)=L_{\text {core }}$ for the calculation of $\chi(r)$ with Eq. (36). In the computations we took the other terms into account properly for the calculation of $L(r)$. For the value of $L_{\mathrm{adv}}$ in the calculation of $L(r)$ we used the following scheme: for the optically thick part we calculated $L_{\text {adv }}$ from Eq. (18) and for the optically thin part we used $L_{\text {adv }}(r) \simeq 2 v(r) L(r) / c$, which is valid if $J \simeq H \simeq K$ (see Eq. (12)). In any case, the exact value of $L_{\mathrm{adv}}$ makes a difference of less than 1 percent in the calculation of $\chi(r)$.

\subsection{The velocity structure}

The density in the supersonic part of the wind, which is needed for the calculation of $\chi$ and $T(r)$, can be expressed in terms of the mass-loss rate and the velocity law. The velocity law in the supersonic part of the wind is approximately a $\beta$-law:

$v(r) \simeq v_{\infty}\left(1-\frac{R_{\mathrm{S}}}{r}\right)^{\beta}$

with the value of $v_{\infty}$ taken from the observations and the value of $\beta$ still to be determined. However, near the sonic point the velocity must deviate from this $\beta$-law, because it would imply that $v\left(R_{\mathrm{S}}\right)=0$, which contradicts the definition of the sonic point. Therefore we adopt a velocity law in the region of $r \geq R_{\mathrm{s}}$ of the type

$$
\begin{aligned}
v(r)= & \left\{w_{1}\left(\frac{R_{\mathrm{S}}}{r}\right)+w_{2}\left(\frac{R_{\mathrm{s}}}{r}\right)^{2}+w_{3}\left(\frac{R_{\mathrm{S}}}{r}\right)^{3}\right\} \\
& +v_{\infty}\left(1-\frac{R_{\mathrm{s}}}{r}\right)^{\beta},
\end{aligned}
$$

with $w_{1}+w_{2}+w_{3}=v_{\mathrm{s}}$. The $\beta$-law is only valid for $r>$ $R_{\mathrm{s}}$. This expression for $v(r)$ is practical for our purpose because (i) the first three terms are small and quickly vanish for $r>R_{\mathrm{S}}$ and (ii) the critial point conditions of Eqs. (26) and (27) directly describe the values of $w_{1}$ and $w_{2}$ with $w_{3}$ adjustable to give $v\left(R_{\mathrm{s}}\right)=v_{\mathrm{s}}$.

We stress that the second part of the expression for $v(r)$, i.e. the $\beta$-law, only enters into our analysis for the calculation of the velocity and density structure in the supersonic part of the wind. For the analysis of the sonic point conditions only the first part is important. This is equivalent to the statement that the $\beta$-law does not start exactly at $R_{\mathrm{s}}$ but at some slightly larger distance where the power-law part slowly merges with the $\beta$-law. (In principle we could have chosen a more elaborate expression for the velocity law that ensures a smooth transition from the subsonic to the supersonic part, than the one adopted here, but that would not have improved the analysis of the sonic point conditions.)

\subsection{The opacity gradient near the sonic point}

If the velocity law and the temperature structure are known, as described above, then the product $\chi(r) L(r)$ can be found from Eq. (36). The solution of the critical point equations, which will give the mass-loss rate, requires knowledge of the gradients of the optical depth and of the radiative luminosity near the sonic point. In normal modeling of a stellar wind one would assume that 
$\chi=f(\rho, T)$ is known and then solve the structure of the wind and find the mass-loss rate. However, as argued in the introduction, the flux-mean opacity in the atmospheres of WR-stars is not known. Therefore, the purpose of our paper is just the opposite: we try to find what the conditions for $\chi$ are that could explain the observed mass-loss rates in terms of optically thick radiation driven winds. A consequence of this approach is that we do not know $\chi(r)$, nor its gradient near the sonic point. Instead, we derive it from the requirement that the model must produce the observed mass-loss rate.

If the gradient of $L(r)$ is known we can derive the gradient of $\chi(r)$ from the derivative of Eq. (36) and vice versa. Therefore we adopt two methods to estimate these gradients: variants $\mathrm{A}$ and $\mathrm{B}$. In the first one we make an assumption about $L(r)$ and find the gradient of $\chi(r)$. In variant $\mathrm{B}$ we make an assumption about the variation of $\chi(r)$ and find the gradient of $L(r)$. In our modeling we will use both variants and show that the results are quite similar. We describe these two variants in more detail in the next section.

\section{The method for calculating optically thick wind models for WR-stars}

We describe the method for solving the equations and for determining the mass-loss rates for optically thick winds of WR-stars. The essence of the analysis is to find under what conditions the optically thick wind models produce the observed mass-loss rates. We will do this for a grid of models of different values of $M_{*}, L_{*}=L_{\text {core }}, R_{\mathrm{s}}$ and $R_{\mathrm{hc}}$.

The adopted scheme consists of choosing the effective optical depth $\tau_{\text {s }}^{\prime}$ at the sonic point. We then make a first estimate of one particular parameter at the sonic point. This parameter is either the radiative luminosity $L_{\mathrm{s}}$ (Variant A) or the opacity $\chi_{\mathrm{s}}$ (Variant B). We then calculate a self-consistent value of $\dot{M}$ and of $v, T, \chi, L$ and their first and second order derivatives at the sonic point, from the above described equations. Knowing these values at the sonic point, we can check if the value of the velocity gradient that is derived from the momentum equation is identical to the one that is needed to satisfy the energy equation. So basically we check that the momentum equation and the energy equation are both satisfied in the transonic region. If this is not the case, we modify our choice of $L_{\mathrm{s}}$ or $\chi_{\mathrm{s}}$. We developed a numerical method that converges very rapidly and gives the self-consistent models within typically about some tens of iterations.

Up to this point we have only considered the conditions at or near the sonic point. The next step is to find the value of the velocity law exponent $\beta$ that is needed to produce the chosen value of $\tau^{\prime}$. This is obtained by integrating the optical depth over the whole wind for different values of $\beta$ until the pre-adopted value of $\tau^{\prime}{ }_{\mathrm{s}}$ is found. The resulting model then gives, for any particular choice of the stellar parameters $M_{*}, L_{*}, R_{\mathrm{s}}, R_{\mathrm{hc}}$ and wind parameter $v_{\infty}$, and for any pre-chosen value of $\tau^{\prime}$ s (which is basically the same as choosing the gas temperature at the sonic point) the resulting value of $\dot{M}$ as well as the temperature and velocity structure of the supersonic part of the wind. The calculated mass-loss rate can then be compared with the observed values.

In the previous section we described two methods for dealing with the opacity: Variants A and B. We discuss the calculation of the models in these two variants separately. In this section we describe the computations for fixed values of $R_{\mathrm{s}}$. These models are named $\mathrm{A} 0$ and B0. In Sect. 8 we will describe the models with a variable value of $R_{\mathrm{s}}$.

\subsection{Variant $A 0$}

In this variant we assume that $L(r)$ is constant in the wind. The variation of the opacity $\chi(r)$ then follows from the condition that the wind is radiatively driven.

First we adopt the values of $R_{\mathrm{s}}$ and $R_{\mathrm{hc}}$ (the range of these parameters for WR-stars will be justified later in Sect. 6.2) and we adopt a value of the effective optical depth $\tau_{\text {s }}^{\prime}$ at this sonic point. We then make a first guess of $L_{\mathrm{s}}$, which is only slightly smaller than $L_{*}$, that sets the value of $T_{\text {eff }}\left(R_{\mathrm{s}}\right)$. This gives the gas temperature at the sonic point $T_{\mathrm{s}}$ (Eq. (34)) as well as the sound speed and the flow velocity, $v_{\mathrm{s}}$. The mass-loss rate $\dot{M}$ then follows from formula (19).

The initial guess values of other terms at the sonic point are found by the scheme:

(i) $\chi_{\mathrm{s}}$ and $(\mathrm{d} T / \mathrm{d} r)_{\mathrm{s}}$ are both found from the combination of Eqs. (16) and (25).

(ii) $(\mathrm{d} v / \mathrm{d} r)_{\mathrm{s}}$ is found from the derivative of the energy equation (10) with the derivative of $L_{\text {adv }}$ (Eq. (18)).

(iii) $\left(\mathrm{d}^{2} T / \mathrm{d} r^{2}\right)_{s}$ and $(\mathrm{d} \chi / \mathrm{d} r)_{\mathrm{s}}$ are found from the combination of Eqs. (16) for the temperature gradient and de l'Hopital's rule (26) for the momentum equation.

(iv) $\left(\mathrm{d}^{2} v / \mathrm{d} r^{2}\right)_{\mathrm{s}}$ is found from the second derivative of the energy Eq. (10).

(v) $\left(\mathrm{d}^{2} \chi / \mathrm{d} r^{2}\right)_{\mathrm{s}}$ and $\left(\mathrm{d}^{3} T / \mathrm{d} r^{3}\right)_{\mathrm{s}}$ are found by combining the regularity condition (Eq. (27)) with the second derivative of the temperature Eq. (16).

(vi) We now have all the required values and gradients at the sonic point and we can test if the momentum and energy equations are both satisfied. To do this we calculate $\mathrm{d} v / \mathrm{d} r$ at a point slightly below the sonic point, e.g. at $r=0.99 R_{\mathrm{s}}$, from the momentum equation by using Taylor expansions for finding $T, \chi$ and $v$ ( $L$ is constant). In this calculation we use the values of these physical terms and their first and second derivatives at the sonic point. $\left(\mathrm{d}^{2} v / \mathrm{d} r^{2}\right)_{\mathrm{s}}$. We then compare this with the value of $\mathrm{d} v / \mathrm{d} r$ at that same point, that is required by the energy equation, i.e. by differentiating Eq. (10). If these two values of $\mathrm{d} v / \mathrm{d} r$ are not the same, our initial guess of the constant value of $L(r)$ was incorrect. We then choose another value of $L(r)$ and repeat the process untill convergence is reached.

(vii) In the last step we calculate the value of $\beta$ in the supersonic part of the wind that gives the adopted value of 
$\tau^{\prime}{ }_{\mathrm{S}}$ (Eq. (35)). We find $\rho(r)$ from the mass continuity equation with the velocity law of Eq. (38), with $w_{1}$ and $w_{2}$ derived from $(\mathrm{d} v / \mathrm{d} r)_{\mathrm{s}}$ and $\left(\mathrm{d}^{2} v / \mathrm{d} r^{2}\right)_{\mathrm{s}}$ and $w_{3}=v_{\mathrm{s}}-w_{1}-w_{2}$. The opacity $\chi(r)$ in the supersonic part of the wind, which is needed for the calculation of $\tau^{\prime}(r)$, is derived from the momentum Eq. (36). The temperature structure in the supersonic part is calculated with Eq. (32) with $\tau^{\prime}(r)$ as input. So in practice we calculate both $\tau^{\prime}(r)$ and $T(r)$ by stepwise integration of the Eq. (33), which results in $T(r)$ through Eq. (32), which gives $\chi(r)$ at the next step through Eq. (36).

We want to point out that only in the final step for the determination of $\beta$ we need a numerical integration. All other steps use straightforward calculations of single values at the critical point. These calculations are exact, insofar as the approximations used to derive the formulae are correct. Therefore the calculations are very fast and the iteration converges rapidly.

\subsection{Variant $B 0$}

In this variant we assume that the opacity can be expressed as $\chi(r)=a \rho / T^{\alpha}$, with $a$ to be determined by the conditions at the sonic point, and $\alpha$ is an adopted constant. For instance, Kramers' opacity law has $\alpha=3.5$. We will adopt several values in the range of $3.0<\alpha<5.0$.

The process of calculating self-consistent optically thick radiation driven wind models is very similar to that used in Variant A0. We again start with stellar parameters $M_{*}, L_{*}, R_{\mathrm{hc}}$ and $R_{\mathrm{s}}$ and we adopt $\tau_{\mathrm{s}}^{\prime}$. As in Variant $\mathrm{A} 0$, we make a first guess of $L_{\mathrm{s}}<L_{*}$. However in Variant B0 $L(r)$ is distance dependent, contrary to the situation in Variant A0. The choice of $L_{\mathrm{s}}$ sets the values of $T_{\mathrm{s}}, v_{\mathrm{s}}$ and $\dot{M}$, as described in Variant A0. The steps for solving the equations at the sonic point are:

(i) $\chi_{\mathrm{s}}$ and $(\mathrm{d} T / \mathrm{d} r)_{\mathrm{s}}$ are both found from the combination of Eqs. (16) and (25). The value of $\chi_{\mathrm{s}}$ then determines the value of $a$ for the adopted opacity dependence.

(ii) $(\mathrm{d} v / \mathrm{d} r)_{\mathrm{s}},(\mathrm{d} L(r) / \mathrm{d} r)_{\mathrm{s}}$ and $\left(\mathrm{d}^{2} T / \mathrm{d} r^{2}\right)_{\mathrm{s}}$ are found from the application of the de l'Hopital's rule (Eq. (26)) combined with the derivatives of the temperature Eq. (16) and the energy Eq. (19). The value of $(\mathrm{d} \chi / \mathrm{d} r)_{\mathrm{s}}$, which is needed in these solutions, is derived from the definition of $\chi=a \rho / T^{\alpha}$ together with $(\mathrm{d} T / d r)_{\mathrm{s}}$ and $(\mathrm{d} v / \mathrm{d} r)_{\mathrm{s}}$.

(iii) $\left(\mathrm{d}^{2} v / \mathrm{d} r^{2}\right)_{\mathrm{s}}$ is found from the regularity condition (Eq. (27)) with $\left(\mathrm{d}^{3} T / \mathrm{d} r^{3}\right)_{\mathrm{s}}$ from the second derivative of the temperature Eq. (16) and $\left(\mathrm{d}^{2} \chi / \mathrm{d} r^{2}\right)_{\mathrm{s}}$ from the adopted expression for $\chi$.

(iv) $(\mathrm{d} L(r) / \mathrm{d} r)_{\mathrm{s}}$ is found from the energy Eq. (19).

(v) We then check if the energy equation and the momentum equation are both satisfied around the sonic point. To do this we calculate $\mathrm{d} v / \mathrm{d} r$ at a point slightly below the sonic point, at $r=0.99 R_{\mathrm{S}}$, from the momentum equation by using for the needed terms $T, v$ and $L$ (with $\chi(r)$ being known) the Taylor expansion formula which uses the values of these physical terms and their first and second derivatives at the sonic point. We then compare this with the value of $\mathrm{d} v / \mathrm{d} r$ at that same point, that is required by the energy equation, i.e. by differentiating Eq. (10). If this is not the case, our initial guess of $L(r)$ was incorrect. We then choose another value of $L(r)$ and repeat the process until convergence is reached.

(vi) When the model has converged, we derive the parameter $\beta$ of the supersonic velocity law that produces the adopted value of $\tau^{\prime}$. This is done in a similar way as in Variant A0, i.e. with stepwise integration of Eq. (33) for $\tau^{\prime}(r)$, with the adopted expression for $\chi(r)$.

\section{Some simple estimates}

In this section we describe some simple estimates, based on general momentum and energy considerations of optically thick radiation driven winds.

\subsection{The opacity at the sonic point}

We can obtain a simple estimate of the opacity at the sonic point of an optically thick wind from the sonic point condition that the righthand side of the momentum equation should vanish, so $f_{1}\left(R_{\mathrm{s}}\right)=0$ with $f_{1}$ defined by Eq. (21). The temperature derivative in an optically thick wind is given by Eq. (16). These equations predict that

$$
\frac{\chi_{\mathrm{s}} L\left(R_{\mathrm{s}}\right)}{4 \pi c}=\frac{G M_{*}-2 v_{\mathrm{s}}^{2} R_{\mathrm{s}}}{1+\left(a_{1} \dot{M}\right) /\left(c_{1} R_{\mathrm{s}}^{2} T_{\mathrm{s}}^{3} v_{\mathrm{s}}\right)}
$$

where we have used the mass continuity equation for the substitution of $\rho$. The second term of the numerator is much smaller than the first term, because the sound velocity at the sonic point (typically about $30 \mathrm{~km} \mathrm{~s}^{-1}$ ) is much smaller than the escape velocity (typically about $2000 \mathrm{~km} \mathrm{~s}^{-1}$ ). For reasonable values of $\dot{M}$ (between $10^{-5}$ and $10^{-4} M_{\odot} \mathrm{yr}^{-1}$ ) and sonic point temperatures of order $10^{5} \mathrm{~K}$ (see below), the second term in the denominator is much smaller than unity. This is equivalent to the statement that the two dominant terms in the right hand side of the momentum equation are the gravity and the radiation pressure, with the gas pressure terms being negligible. So Eq. (39) reduces to

$\chi_{\mathrm{s}} \simeq \frac{4 \pi c G M_{*}}{L_{*}}$

Using the mass-luminosity relation of H-poor WR-stars, Eq. (53), we find an estimate of the required opacity for radiation driven optically thick winds of $0.3<\chi_{\mathrm{s}}<$ $0.9 \mathrm{~cm}^{2} \mathrm{~g}^{-1}$. These are reasonable values for hot star winds.

\subsection{The opacity gradient at the sonic point}

We can derive an important condition for the opacity gradient at the sonic point of radiation driven winds. From de l'Hopital's rule at the sonic point (Eq. (26)), combined with the sonic point condition that $f_{1}=0$ and $f_{2}=0$, 
it is easy to show that the velocity gradient at the sonic point, $(\mathrm{d} v / \mathrm{d} r)_{\mathrm{s}}$, can only be positive if

$$
\left(\frac{\mathrm{d} \chi}{\mathrm{d} r}\right)_{\mathrm{s}}+\frac{\chi_{\mathrm{s}}}{L_{\mathrm{s}}}\left(\frac{\mathrm{d} L}{\mathrm{~d} r}\right)_{\mathrm{s}}+\frac{4 \pi c a_{1}}{L_{\mathrm{s}}}\left(2 T_{\mathrm{s}}-R_{\mathrm{s}}^{2}\left(\frac{\mathrm{d}^{2} T}{\mathrm{~d} r^{2}}\right)_{\mathrm{s}}\right)>0
$$

The last term is due to the effect of the gradient of the gas pressure. In a radiation driven wind the force produced by the gas pressure is by definition negligible compared to the force produced by the radiation pressure. So we can neglect that term. Equation (41) thus shows that in a radiation driven wind with constant $L(r)$ the gradient $(\mathrm{d} \chi / \mathrm{d} r)_{\mathrm{s}}$ must be positive to allow a transonic flow. In the case of optically thick radiation driven winds $\mathrm{d} L(r) / \mathrm{d} r \leq$ 0 (cf. with Quinn \& Paczyński 1985) and so $(\mathrm{d} \chi / \mathrm{d} r)_{\mathrm{s}}$ must certainly be positive.

We conclude that a stellar wind can only be driven by radiation pressure if $\mathrm{d} \chi / \mathrm{d} r>0$ at the sonic point! This implies that optically thick stellar winds must have their sonic point in the density and temperature regime where the opacity increases as a function of distance. This occurs for instance in the region of the iron opacity peak around $\log T \simeq 5.2$.

This condition for the gradient of the opacity of radiation driven winds is a special case of the general condition that a wind can only be accelerated through the sonic point if either energy or momentum is added at the sonic point (cf. Lamers \& Cassinelli 1999, p. 100).

\subsection{Mass-loss rates of optically thick winds}

The energy equation near the sonic point (Eq. (19)) can be written as

$$
\frac{G M \dot{M}}{R_{\mathrm{s}}} \simeq L_{\mathrm{adv}}-\left\{\dot{E}-L\left(R_{\mathrm{s}}\right)\right\}
$$

The test models and the optically thick wind models of the studied stars (described below in Sect. 5.1 and Table 1) reveal that $L\left(R_{\mathrm{S}}\right)$ is very close to $\dot{E}$ and the absolute value of $L\left(R_{\mathrm{s}}\right)-\dot{E}$ is much smaller than $L_{\mathrm{adv}}\left(R_{\mathrm{s}}\right)$. This means that we can estimate the mass-loss rate by equating the advection term with the potential energy term:

$$
\frac{G M \dot{M}}{R_{\mathrm{s}}}=L_{\mathrm{adv}}\left(R_{\mathrm{s}}\right)
$$

and after some substitutions we find

$$
\dot{M} \simeq \frac{c_{1} R_{\mathrm{s}}^{3} T_{\mathrm{s}}^{4} v_{\mathrm{s}}}{G M}=\frac{c_{1} a_{1}^{1 / 2} R_{\mathrm{s}}^{3} T_{\mathrm{s}}^{4.5}}{G M}
$$

Approximately the same formula can be derived quite generally from the following simple considerations. Let us start with the formulae for $T_{\mathrm{s}}$ and $\tau_{\mathrm{s}}^{\prime}$ (Eqs. (34) and (35)). Using Eq. (4) we can express $\tau_{\mathrm{s}}^{\prime}$ in the form

$\tau_{\mathrm{s}}^{\prime}=\int_{R_{\mathrm{s}}}^{\infty} \frac{\chi \dot{M} R_{\mathrm{s}}^{2}}{4 \pi v r^{4}} \mathrm{~d} r$

The ratio $\chi / v$ is changing very little in the inner part of the wind (this is the specific property of optically thick winds of WR-stars!) and the ratio $\chi / v$ at infinity is only about two times smaller than at the sonic point. This property and the steep drop of the integral kernel in Eq. (45) with increasing $r$, due to the factor $v^{-1} r^{-4}$, implies that we can estimate $\tau_{\mathrm{s}}^{\prime}$ as

$\tau_{\mathrm{s}}^{\prime} \approx \frac{c_{\mathrm{m}} \chi_{\mathrm{s}}}{v_{\mathrm{s}}} \frac{\dot{M}}{4 \pi R_{\mathrm{s}}}$

where $c_{\mathrm{m}}$ is a multiplier of the order of unity. The temperature at the sonic point can be expressed approximately as

$T_{\mathrm{s}}^{4} \approx \frac{0.75 L_{\mathrm{s}}}{4 \pi \sigma R_{\mathrm{s}}^{2}} \tau_{\mathrm{s}}^{\prime}$

because $\tau_{\mathrm{s}}^{\prime} \gg 2 / 3$ for WR-winds. Using Eqs. (47) and (46) and $\chi_{\mathrm{s}}$ from Eq. (40), we obtain

$\dot{M} \simeq \frac{a_{1}^{1 / 2} c_{1} R_{\mathrm{s}}^{3} T_{\mathrm{s}}^{4.5}}{G M} \frac{0.75}{c_{\mathrm{m}}}$.

Note that this formula differs from the Eq. (44) only by the constant $0.75 / c_{\mathrm{m}}$ which is very close to unity.

Therefore we can conclude that formula (44) is a very good approximation formula for deriving the mass-loss rates of WR-stars.

\subsection{The minimum mass-loss rate for optically thick winds}

We can find the minimum estimated mass-loss rate for the particular WR-star by using the formula (44) with the minimim estimate of the temperature at the sonic point derived from formula Eq. (34) by adopting $\tau_{\mathrm{s}}=0$. This gives a minimum value of the mass-loss rate of

$\dot{M}_{\min } \simeq \frac{a_{1}^{1 / 2} c_{1}}{(8 \pi)^{9 / 8}} \frac{R_{\mathrm{s}}^{3 / 4} L_{*}^{9 / 8}}{G M \sigma^{9 / 8}}$.

Applying this equation to a typical hydrogen-free WNEstar with $M_{*} \simeq 10 M_{\odot}, R_{\mathrm{s}} \simeq 2 R_{\odot}$ and with the luminosity given by the $M-L$ law (Eq. (53): $L_{*}=$ $\left.1.85 \times 10^{5} L_{\odot}\right)$, we find a minimum mass-loss rate of about $0.2 \times 10^{-6} M_{\odot} \mathrm{yr}^{-1}$.

\subsection{The maximum mass-loss rate}

An absolute upper limit for the mass-loss rate of radiation driven winds is set by the condition that all the energy generated in the nucleus is used to drive the wind. This gives

$\dot{M}<L /\left(v_{\infty}^{2} / 2+G M / R_{\mathrm{hc}}\right)$.

For a typical hydrogen-free WNE-star with $M_{*} \simeq 10 M_{\odot}$, $v_{\infty}=2000 \mathrm{~km} \mathrm{~s}^{-1}$ and with the luminosity given by the $M-L$ law (Eq. (53): $L_{*}=1.85 \times 10^{5} L_{\odot}$ ) and the hydrostatic core radius given by the evolutionary models (Eq. (54): $\left.R_{\mathrm{hc}} \simeq 0.86 R_{\odot}\right)$ we derive an upper limit of $2.7 \times 10^{-4} M_{\odot} \mathrm{yr}^{-1}$. 
A more realistic upper limit can be found from the formula Eq. (43) by using for $L_{\mathrm{adv}}\left(R_{\mathrm{s}}\right)$ the advective luminosity at infinity which ought to be higher than $L_{\mathrm{adv}}\left(R_{\mathrm{S}}\right)$. In the case of thin winds we know that near the star $L_{\mathrm{adv}} \approx v / c L(r)$, which is much lower than $L_{\mathrm{adv}}(\infty)=$ $2 v_{\infty} L(\infty) / c$. In the case of thick winds we know that $L_{\text {adv }}(\infty) \geq L_{\text {adv }}\left(R_{\mathrm{s}}\right)$. So the upper limit for the massloss rate of optically thick winds can be obtained from the formula

$\dot{M}_{\max } \simeq \frac{2 L(\infty) v_{\infty} R_{\mathrm{s}}}{c G M}$.

For a typical WNE-star with the parameters given above we find that $\dot{M}_{\max }=1.6 \times 10^{-5} M_{\odot} \mathrm{yr}^{-1}$. The observed mass-loss rates of WNE stars are indeed in between the minimum and maximum values derived here.

\section{Test models of optically thick radiation driven winds}

In this section we describe some of the results of the optically thick radiation driven winds of some test models. We discuss the properties of the models and investigate the dependence of the resulting mass-loss rates on the input parameters. We do this for the models calculated in Variant A0 (constant $L(r)$ ) and variant B0 (power-law dependence of $\chi$ ). These tests are useful for describing and understanding the models, and for comparing the results for different parameters.

For the stellar parameters of the test model we have chosen the values of a characteristic WN 5 star: WR139. This star is a member of a well studied binary system and its distance and stellar parameters are well determined (Nugis et al. 1998; Nugis \& Lamers 2000). The adopted parameters are discussed below in Sect. 6 and listed in Table 4 . The luminosity is $1.62 \times 10^{5} L_{\odot}$, the observed mass-loss rate is $0.92 \times 10^{-5} M_{\odot} \mathrm{yr}^{-1}$ and the terminal velocity of the wind is $1785 \mathrm{~km} \mathrm{~s}^{-1}$. The adopted radius of the sonic point is $R_{\mathrm{S}}=2 R_{\mathrm{evol}}=1.648 R_{\odot}$. This is much smaller than the empirically determined "core"-radius of the star, $R_{\mathrm{c}} \simeq 6 R_{\odot}$, derived from the empirical wind models with an adopted $\beta=1$ law by Hamann \& Schwarz (1992). The reason for this smaller choice will be justified below in Sect. 6. Note that for the final set of optically thick wind models (Variants A1 and B1) we will determine the sonic point radius from the demand that $\mathrm{d} \chi / \mathrm{d} r$ is equal to the OPAL value for the particular density, temperature and chemical composition.

\subsection{Test models with a fixed mass-loss rate}

Here we describe the results of a set of models that were calculated with a fixed, pre-specified mass-loss rate. For these models $R_{\mathrm{S}}$ is fixed and the effective optical depth at the sonic point is a parameter that has to be solved. The results of the test models are listed in Table 1 . The first part gives the results of Variant B0, i.e. for a power-law opacity of the type $\chi(r)=a \rho / T^{n}$, for various values of $n$.
The last line gives the results for Variant A0, i.e. with a fixed value of $L(r)$. We discuss some properties of the models.

The value of the optical depth $\chi_{\mathrm{s}}$ at the sonic point is similar for all models. This was already predicted in the previous section where we showed that the gravity force and the radiative force should cancel each other at the sonic point (Eqs. (39) and (40)). All models show that the temperature at the sonic point is about $1.8 \times 10^{5} \mathrm{~K}$. The value of $\tau_{\mathrm{s}}^{\prime}$ needed to reach this temperature is about 20. The high sonic temperature is needed because the mass-loss rate is basically determined by the energy conservation at the sonic point. The velocity gradient implies a gain in potential and kinetic energy that has to be provided under the constraint of constant total energy. For the pre-chosen mass-loss rate this can only be achieved at high temperature, and hence at high effective optical depth. The optical depth of order 20 at the sonic point, with the given mass-loss rate and terminal velocity, requires a high column density above the sonic point, which implies a high value of the velocity law exponent $\beta$ of about 5 in the supersonic part of the wind for most models. The models with high values of $n \geq 4$ require a larger value of $\beta$ because the opacity drops steeply outwards and so the density $\rho \sim\left(r^{2} v\right)^{-1}$ has to drop slowly outwards, implying a high value of $\beta$. In fact there is an upper limit for the opacity exponent $n$ in our models. If $n \geq 4.7$ the opacity decreases so rapidly outwards that the required value of $\tau^{\prime}{ }_{\mathrm{s}} \simeq 20$ cannot be reached by any $\beta$-type velocity law. The slowest velocity law with $\beta \rightarrow \infty$ still has a density decrease of $\rho \sim r^{-2}$ and so the maximum column density above the sonic point is $\dot{M} / 4 \pi R_{\mathrm{s}} v_{\mathrm{s}}$. We also found that there is a lower limit for the exponent $n$ of the opacity law that can produce optically thick radiation driven wind models. If $n<3$ then the opacity does not increase through the sonic point, so the wind cannot be accelerated through the sonic point. We conclude that optically thick radiation driven winds for WR-stars with an opacity of the type $\chi \sim \rho / T^{n}$ near the sonic point can only exist for a small range of $n$ of approximately $3<n<5$.

The data in Table 1 show that the radiative luminosity in the comoving frame is very close to the total luminosity $L_{*}$. The difference is less than about a factor $10^{-2}$. This justifies our assumption that in the calculation of the radiative acceleration (Eq. (17)) we can substitute $L_{*}$ for $L(r)$. Note that in models with variant B0 the value of $L\left(R_{\mathrm{s}}\right)$, that is calculated from the energy Eq. (19) is larger than the value of $\dot{E}$, but less than $L_{*}$. In the case of Variant A0, which has a constant $L(r)$, the comoving luminosity is slightly smaller than $\dot{E}$ but again the difference is very small.

It is interesting to compare the advected luminosity with the difference between $\dot{E}$ and $L\left(R_{\mathrm{s}}\right)$. We see that in all these models the advective luminosity is much larger than this difference. So at the critical point, the energy balance (Eq. (19)) is practically reduced to $L_{\mathrm{adv}}=$ $G M \dot{M} / R_{\mathrm{s}}$. (This property was already used to derive an estimate for mass-loss rate in Eq. (44).) 
Table 1. Test models for WR139 for fixed mass-loss rate.

\begin{tabular}{|c|c|c|c|c|c|c|c|c|c|}
\hline$(1)$ & $\begin{array}{l}\tau_{\mathrm{s}}^{\prime} \\
(2) \\
\end{array}$ & $\begin{array}{c}T_{\mathrm{s}} \\
10^{5} \mathrm{~K} \\
(3) \\
\end{array}$ & $\begin{array}{c}\left(\frac{\mathrm{d} \chi}{\mathrm{d} r}\right)_{\mathrm{s}} \\
10^{-14} \mathrm{~cm} \mathrm{~g}^{-1} \\
(4)\end{array}$ & $\begin{array}{c}v_{\mathrm{s}} \\
\mathrm{km} \mathrm{s}^{-1} \\
(5)\end{array}$ & $\begin{array}{c}\frac{R_{\mathrm{S}}}{v_{\mathrm{s}}}\left(\frac{\mathrm{d} v}{\mathrm{~d} r}\right)_{\mathrm{s}} \\
(6)\end{array}$ & $\begin{array}{c}\frac{\dot{E}-L\left(R_{\mathrm{S}}\right)}{\dot{E}} \\
10^{-4} \\
(7) \\
\end{array}$ & $\begin{array}{c}\frac{L_{\mathrm{adv}}\left(R_{\mathrm{S}}\right)}{\dot{E}} \\
10^{-4} \\
(8)\end{array}$ & $\begin{array}{c}\chi_{\mathrm{s}} \\
\mathrm{cm}^{2} \mathrm{~g}^{-1} \\
(9)\end{array}$ & $(10)$ \\
\hline 3.01 & 21.2 & 1.809 & 2.34 & 34.75 & 1.01 & -0.233 & 101 & 0.756 & 5.09 \\
\hline 3.10 & 20.7 & 1.799 & 3.72 & 34.65 & 1.18 & -2.829 & 98.6 & 0.756 & 5.00 \\
\hline 3.30 & 19.8 & 1.781 & 6.97 & 34.48 & 1.54 & -7.128 & 94.3 & 0.756 & 4.95 \\
\hline 3.50 & 19.2 & 1.768 & 10.45 & 34.35 & 1.88 & -10.21 & 91.25 & 0.755 & 5.03 \\
\hline 4.00 & 18.2 & 1.746 & 20.20 & 34.14 & 2.68 & -15.23 & 86.25 & 0.755 & 5.85 \\
\hline 4.50 & 17.6 & 1.732 & 31.36 & 34.00 & 3.44 & -18.30 & 83.21 & 0.755 & 10.0 \\
\hline 4.70 & 17.5 & 1.727 & 36.19 & 33.96 & 3.74 & -19.20 & 82.25 & 0.755 & 80.0 \\
\hline- & 22.6 & 1.837 & 1.45 & 35.02 & 0.805 & +7.013 & 108.5 & 0.757 & 5.92 \\
\hline
\end{tabular}

The upper part of the table is for Variant B0, the last line forVariant A0.

Table 2. Test models for WR139 with variable mass-loss rate.

\begin{tabular}{rccc}
\hline \hline$\tau_{\mathrm{s}}^{\prime}$ & $\begin{array}{c}\dot{M} \\
10^{-5}\end{array} M_{\odot} \mathrm{yr}^{-1}$ & $\begin{array}{c}T_{\mathrm{s}} \\
10^{5} \mathrm{~K}\end{array}$ & $\beta$ \\
\hline 2.0 & 0.098 & 1.074 & 2.89 \\
3.0 & 0.140 & 1.163 & 3.32 \\
5.0 & 0.228 & 1.296 & 3.87 \\
10.0 & 0.462 & 1.516 & 4.56 \\
20.0 & 0.960 & 1.784 & 5.05 \\
30.0 & 1.477 & 1.964 & 5.25 \\
50.0 & 2.531 & 2.214 & 5.38 \\
\hline \multicolumn{4}{l}{ Models with $\chi \sim \rho / T^{3.5}}$.
\end{tabular}

Models with $\chi \sim \rho / T^{3.5}$.

\subsection{Test models with different mass-loss rates}

For this set of test models we have solved the full set of equations described above, for calculating the mass-loss rate of optically thick radiation driven wind models. In these calculations the mass-loss rate is determined basically from the predicted velocity gradient near the sonic point which is required by the sonic point conditions. The velocity gradient implies a potential and kinetic energy gain that has to be provided under the constraint of constant total energy. Table 2 gives the results for Variant B0, i.e. with an opacity-law of the form $\chi=a \rho / T^{n}$ for $n=3.5$ and with fixed $R_{\mathrm{s}}=2 R_{\text {evol }}$. In this case we have adopted a pre-chosen set of values for $\tau^{\prime}$ s and we derive the mass-loss rate. We see that both the mass-loss rate and the temperature at the sonic point increase with increasing effective optical depth. This is in agreement with the predictions described in the approximate formulae Eqs. (46) and (47). For higher optical depth the velocity laws in the supersonic part of the wind have to become "softer", i.e. higher values of $\beta$, to have a sufficient column density. We see that the observed mass-loss rate of $\dot{M}=0.92 \times 10^{-5} M_{\odot} \mathrm{yr}^{-1}$ requires an effective optical depth at the sonic point of about 20, in agreement with the results of Table 1.

Figure 1 shows the mass-loss rates and the values of $\beta$ for a series of test models for WR139, for different values

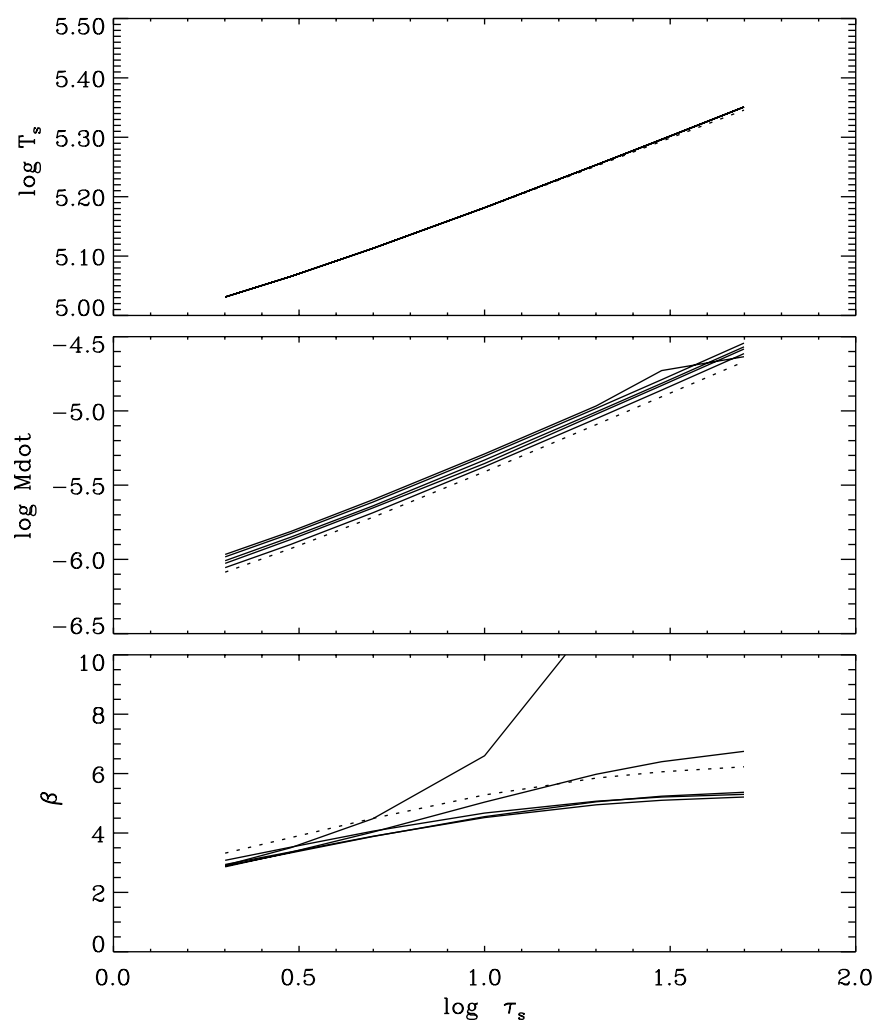

Fig. 1. The results of models B0, i.e. for $\chi \sim \rho / T^{n}$, for the star WR139 (WN5). The temperature $T_{\mathrm{s}}$ at the sonic point (upper), the mass-loss rate (middle) and the value of $\beta$ (lower) are plotted as a function of the effective optical depth $\tau_{\mathrm{s}}^{\prime}$ at the sonic point. The curves are for $n=3.01,3.30,3.50,4.0$ and 4.5. In all plots the lowest curve is for $n=3.01$ and the highest one is for $n=4.50$. Notice that the results are insensitive to the value of $n$, except the value of $\beta$ for $n>3.5$. The dotted line is for model A0, i.e. for $L(r)=$ constant in the transonic region. The results for models $\mathrm{A} 0$ and $\mathrm{B} 0$ are very similar.

of $n$. We see that the mass-loss rate scales almost linearly with the adopted value of $\tau^{\prime}$ s as it is expected according to the approximate formula (46). The mass-loss rates are not very sensitive to the values of $n$. 


\subsection{The influence of the sonic radius}

In this section we study the influence of the sonic radius on the mass-loss rates of WR-stars. We also investigate the influence of the choices for determining $\chi(r)$ (Variants A0 or $\mathrm{B} 0)$ on the mass-loss rates, predicted for the models. Table 3 gives the results for a series of test models for WR139, for various values of the ratio $R_{\mathrm{S}} / R_{\text {evol }}$ and for different values of $\tau^{\prime}{ }_{\mathrm{s}}$. We give the results for both variants (A0 and $\mathrm{B} 0$ ). The values of the sonic point temperature $T_{\mathrm{s}}$ are slightly different for Variants A0 and B0. However, this difference is so small, less than a factor $10^{-3}$, that we only listed the values of $T_{\mathrm{s}}$ for variant $\mathrm{A} 0$. We see that the results of the two variants are quite similar. The difference in the mass-loss rates and in $\beta$ is less than about 25 percent, with mass-loss rates of Variant B0 being slightly higher than those of Variant A0. The values of $\beta$ of Variant B0 are slightly smaller than those of Variant A0. This is because Variant B0 has higher mass-loss rates, so a slightly steeper velocity law is needed to produce the same value of $\tau^{\prime}{ }_{\mathrm{s}}$. The mass-loss rates increase with increasing adopted location of the sonic point. In the range of $1<R_{\mathrm{s}} / R_{\text {evol }}<4$ the mass-loss rate for a fixed value of $\tau^{\prime}{ }_{\mathrm{s}}$ increases almost linearly with $R_{\mathrm{s}}$. This is in accord with the prediction of the approximate formula (46).

\subsection{Conclusions from the tests}

We conclude from these tests that the mass-loss rates predicted by optically thick radiation driven wind models show the following properties:

1. The high mass-loss rates of the star WR139 requires a high temperature at the sonic point of order $10^{5} \mathrm{~K}$, depending on the adopted radius of the sonic point. This high temperature then implies a considerable effective optical depth, $\tau^{\prime}{ }_{\mathrm{s}} \simeq 20$. The large optical depth requires a slow velocity law in the supersonic part of the wind with $\beta \approx 5$. In the discussion, Sect. 9, we will show that there is indeed observational evidence for such a high value of $\beta$.

2. The mass-loss rate of the WR139 models (or more generally of models with fixed $L, M, v_{\infty}$ and chemical composition) scales almost linearly with the adopted value of the sonic radius, with the optical depth and with the square root of the sonic point temperature, i.e. $\dot{M} \sim R_{\mathrm{s}} \tau^{\prime}{ }_{\mathrm{s}} T_{\mathrm{s}}^{1 / 2}$. The temperature at the sonic point scales as $T_{\mathrm{s}} \sim R_{\mathrm{s}}^{-1 / 2} \tau_{\mathrm{s}}^{\prime 1 / 4}$. Combining these two relations we find that

$\dot{M} \sim T_{\mathrm{s}}^{4.5} R_{\mathrm{s}}^{3}$.

This is in accord with the prediction of Eq. (44). We can conclude that optically thick wind models (A0 and B0) for a particular WR-star lead to the same mass-loss rate for different combinations of parameters $T_{\mathrm{s}}$ and $R_{\mathrm{s}}$ that satisfy the formula (52). This conclusion means that for a particular star we can get the estimate of $R_{\mathrm{s}}$ by demanding that $T_{\mathrm{s}}$ must be such
Table 3. Test models for WR139 with variable sonic point radius.

\begin{tabular}{|c|c|c|c|c|c|c|c|}
\hline $\begin{array}{c}R_{\mathrm{s}} \\
R_{\mathrm{evol}}\end{array}$ & $\begin{array}{c}R_{\mathrm{hc}} \\
R_{\mathrm{evol}}\end{array}$ & $\tau_{\mathrm{s}}^{\prime}$ & $\begin{array}{c}T_{\mathrm{s}} \\
\mathrm{K} \\
\mathrm{A} 0\end{array}$ & $\dot{M}^{1}$ & $\dot{M}^{1}$ & $\mathrm{~A} 0$ & B0 \\
\hline 1.0 & 1.0 & 2.0 & 152000 & 0.049 & 0.058 & 3.22 & 2.81 \\
\hline 1.0 & 1.0 & 3.0 & 164500 & 0.070 & 0.083 & 3.72 & 3.23 \\
\hline 1.0 & 1.0 & 5.0 & 183400 & 0.114 & 0.136 & 4.35 & 3.77 \\
\hline 1.0 & 1.0 & 10.0 & 214700 & 0.232 & 0.276 & 5.12 & 4.45 \\
\hline 1.0 & 1.0 & 20.0 & 252900 & 0.485 & 0.576 & 5.67 & 4.94 \\
\hline 1.0 & 1.0 & 30.0 & 278700 & 0.750 & 0.891 & 5.88 & 5.14 \\
\hline 1.0 & 1.0 & 50.0 & 314900 & 1.301 & 1.543 & 6.05 & 5.28 \\
\hline 2.0 & 1.0 & 2.0 & 107400 & 0.082 & 0.098 & 3.32 & 2.89 \\
\hline 2.0 & 1.0 & 3.0 & 116300 & 0.118 & 0.140 & 3.84 & 3.32 \\
\hline 2.0 & 1.0 & 5.0 & 129600 & 0.192 & 0.228 & 4.49 & 3.87 \\
\hline 2.0 & 1.0 & 10.0 & 151700 & 0.389 & 0.462 & 5.28 & 4.56 \\
\hline 2.0 & 1.0 & 20.0 & 178500 & 0.809 & 0.960 & 5.85 & 5.05 \\
\hline 2.0 & 1.0 & 30.0 & 196500 & 1.247 & 1.477 & 6.06 & 5.25 \\
\hline 2.0 & 1.0 & 50.0 & 221700 & 2.143 & 2.531 & 6.23 & 5.38 \\
\hline 4.0 & 2.0 & 2.0 & 76000 & 0.138 & 0.165 & 3.40 & 2.94 \\
\hline 4.0 & 2.0 & 3.0 & 82300 & 0.198 & 0.235 & 3.93 & 3.37 \\
\hline 4.0 & 2.0 & 5.0 & 91700 & 0.323 & 0.384 & 4.59 & 3.93 \\
\hline 4.0 & 2.0 & 10.0 & 107300 & 0.654 & 0.778 & 5.40 & 4.61 \\
\hline 4.0 & 2.0 & 20.0 & 126300 & 1.365 & 1.620 & 5.97 & 5.08 \\
\hline 4.0 & 2.0 & 30.0 & 139100 & 2.108 & 2.499 & 6.19 & 5.26 \\
\hline 4.0 & 2.0 & 50.0 & 157000 & 3.635 & 4.298 & 6.35 & 5.37 \\
\hline 4.0 & 1.0 & 2.0 & 75900 & 0.138 & 0.164 & 3.40 & 2.94 \\
\hline 4.0 & 1.0 & 3.0 & 82200 & 0.198 & 0.235 & 3.93 & 3.37 \\
\hline 4.0 & 1.0 & 5.0 & 91600 & 0.321 & 0.382 & 4.59 & 3.93 \\
\hline 4.0 & 1.0 & 10.0 & 107100 & 0.649 & 0.770 & 5.40 & 4.61 \\
\hline 4.0 & 1.0 & 20.0 & 125900 & 1.343 & 1.589 & 5.97 & 5.09 \\
\hline 4.0 & 1.0 & 30.0 & 138300 & 2.055 & 2.425 & 6.19 & 5.27 \\
\hline 4.0 & 1.0 & 50.0 & 155500 & 3.481 & 4.086 & 6.36 & 5.39 \\
\hline
\end{tabular}

${ }^{1} \dot{M}$ is in units of $10^{-5} M_{\odot} \mathrm{yr}^{-1}$.

that the OPAL opacity gradient from OPAL Tables is positive. We will show below in Sect. 8 that this occurs at $T_{\mathrm{s}} \geq 156000 \mathrm{~K}$ or $37000<T_{\mathrm{s}}<71000 \mathrm{~K}$.

3. The models with the two variants $\mathrm{A} 0$ and $\mathrm{B} 0$ for the opacity give about the same mass-loss rates and the same values of $\beta$ with a difference less than about 25 percent. This gives confidence in the results that we will obtain below for the modeling of the individual WR-stars.

4. The results are insensitive to the choice of the parameter $R_{\mathrm{hc}}$, as can bee seen by comparing the models of $R_{\mathrm{s}} / R_{\text {evol }}=4$ with $R_{\mathrm{hc}} / R_{\text {evol }}=2.0$ or 1.0 (and confirmed with other test calculations). So a possible uncertainty in the value of the parameter $R_{\mathrm{hc}}$ has only a minor effect on the resulting model.

\section{Parameters of typical WR-stars}

Up to this point we have considered the properties of optically thick radiation driven wind models and their 
Table 4. Parameters of WR-stars used for modeling the optically thick winds.

\begin{tabular}{rlrrrlllll}
\hline \hline Star & Sp. Type & $\begin{array}{c}\log L \\
(L \odot)\end{array}$ & $\begin{array}{c}M_{\mathrm{WR}} \\
\left(M_{\odot}\right)\end{array}$ & $\begin{array}{l}R_{\text {evol }} \\
\left(R_{\odot}\right)\end{array}$ & $Y$ & $Z$ & $\begin{array}{c}\dot{M}(\mathrm{obs}) \\
\left(10^{-5}\right) \\
\left(M_{\odot} \mathrm{yr}^{-1}\right)\end{array}$ & $\begin{array}{c}v_{\infty} \\
\left(\mathrm{km} \mathrm{s}^{-1}\right)\end{array}$ & $\begin{array}{c}\frac{\dot{M} v_{\infty}}{\eta / c} \\
\eta\end{array}$ \\
\hline 2 & WN 2b & 5.27 & 10.0 & 0.863 & 0.983 & 0.0172 & 0.40 & 3100 & 3.29 \\
139 & WN 5+O 6V & 5.21 & 9.3 & 0.824 & 0.936 & 0.0172 & 0.92 & 1785 & 4.96 \\
136 & WN 6b & 5.73 & 19.1 & 1.235 & 0.866 & 0.0173 & 6.25 & 1605 & 9.20 \\
22 & WN 7+OB & 6.08 & 55.3 & 1.622 & 0.546 & 0.0176 & 4.20 & 1790 & 3.06 \\
105 & WN 9 & 5.81 & 21.8 & 1.315 & 0.624 & 0.0176 & 2.80 & 1200 & 2.54 \\
\hline 111 & WC 5 & 5.31 & 10.6 & 0.891 & 0.381 & 0.619 & 1.00 & 2415 & 5.82 \\
42 & WC 7+O 7V & 5.23 & 9.5 & 0.837 & 0.497 & 0.503 & 1.28 & 1645 & 6.11 \\
103 & WC 9 & 5.20 & 9.2 & 0.818 & 0.585 & 0.415 & 2.40 & 1190 & 8.75 \\
\hline
\end{tabular}

dependence on the input parameters. In the next two sections 6 , and 7 , we describe models for a series of WR-stars of different types. For the study of the mechanisms for the triggering and driving of the wind, we have to know the typical stellar and wind parameters of WR-stars. We will use a few well-studied WR-stars for the detailed analysis of the optically thick wind models.

\subsection{Masses, luminosities and radii}

For the detailed analysis we will use the Galactic WRstars with well determined stellar and mass-loss parameters from Nugis \& Lamers (2000). Table 4 presents the selected stars and their parameters. The table gives the spectral types, luminosities, masses, chemical composition of the atmospheres, mass-loss rates and terminal velocities of the winds. The masses are derived from the luminosities, using the predicted mass-luminosity relation by Schaerer \& Maeder (1992)

$\log \frac{L}{L_{\odot}}=3.032+2.695 \log \frac{M}{M_{\odot}}-0.461\left(\log \frac{M}{M_{\odot}}\right)^{2}$.

The radius of the hydrostatic core, $R_{\text {evol, }}$ is from the evolutionary calculations by Schaerer \& Maeder (1992). They derived the following relation between luminosity and radius

$\log \frac{R_{\text {evol }}}{R_{\odot}}=-1.845+0.338 \log \frac{L}{L_{\odot}}$.

The last column gives the value of the momentum transfer efficiency

$\eta=\dot{M} v_{\infty} /(L / c)$

These values are between about 2 and 10 .

\subsection{The radius of the sonic point, $R_{\mathrm{s}}$}

One of the key parameters of our models is the radius of the sonic point, $R_{\mathrm{s}}$. To get a reasonable estimate of this value for WR-stars, we discuss the results of the empirical studies.
Detailed spectroscopic non-LTE modeling of the winds of WR-stars by Koesterke \& Hamann (1995), Hamann \& Koesterke (1998a,1998b) and Dessart et al. (2000) results in an estimate of the "core-radius" (here referred to as $R_{\tau 20}$ ), which is the inner boundary of their model atmospheres. The core radius is one of the basic parameters that specify the model atmosphere and is defined by either at Rosseland optical depth of 20 or at a small specified wind velocity (about $1 \mathrm{~km} \mathrm{~s}^{-1}$ ).

The core radii can be compared with the hydrostatic radii, $R_{\text {evol }}$, of the evolutionary models. This is shown in Table 5. We separately give the results of modeling the WR-stars with the "standard models" used by Koesterke \& Hamann (1995) and Hamann \& Koesterke (1998a, $1998 b)$, and those with the new models by Dessart et al. (2000). These last models for WC-stars are more realistic, as they take into account blanketing and clumping. We see that the ratio $R_{\tau 20} / R_{\text {evol }}$ increases from about 2 for earlyWN stars to about 20 for late-WN stars, and from about 2 to $5-10$ from early-WC to late-WC stars. Although $R_{\tau 20}$ is not the same as the radius $R_{\mathrm{s}}$ of sonic point, we can expect that $R_{\mathrm{s}} / R_{\text {evol }}$ also increases towards later spectral types.

Studies of the light-curves of eclipsing and spectroscopic binaries with WR-components indicate that the photospheric radius $R_{\text {phot }}$ at $\tau \approx 1$ is smaller than the core radius $R_{\tau 20}$ derived from the spectrum by using the "standard models". See Cherepashchuk (1991, 2000) for V444 Cyg and CQ Cep. This is due to the fact that the standard models have a too steep velocity law of $\beta=1$, whereas the true velocity law is less steep (see Sect. 9). The difference between $R_{\tau 20}$ and $R_{\text {phot }}$ amounts to about a factor 2 . At the photospheric radius the wind velocity is several hundred $\mathrm{km} \mathrm{s}^{-1}$ (Cherepashchuk 1991, 2000), and so the sonic radius, where $v \approx 30 \mathrm{~km} \mathrm{~s}^{-1}$ must be even smaller. These arguments show that the sonic radius of the WR-winds is uncertain, but is probably on the order of $0.5 R_{\tau 20}$ for early subtypes and about $R_{\tau 20}$ for late subtypes. The values of $R_{\mathrm{s}} / R_{\text {evol }}$ adopted as a first guess of $R_{\mathrm{s}}$ as a function of spectral type are listed in the last column of Table 5 , with the values of $R_{\text {evol }}$ given in Table 4 . If the ratio $R_{\mathrm{S}} / R_{\text {evol }}$ is very uncertain, as indicated by a range 
Table 5. Core radii $R_{\tau 20}$ and the adopted sonic radii $R_{\mathrm{s}}$.

\begin{tabular}{l|cr|cr|c}
\hline \hline Subclass & $\begin{array}{c}R_{\tau 20} \\
\left(R_{\text {evol }}\right)\end{array}$ & $\begin{array}{r}\text { Nr } \\
\text { Stars }\end{array}$ & $\begin{array}{c}R_{\tau 20} \\
\left(R_{\text {evol }}\right)\end{array}$ & $\begin{array}{r}\text { Nr } \\
\text { Stars }\end{array}$ & $\begin{array}{c}R_{\mathrm{s}} / R_{\text {evol }} \\
\text { adopted }\end{array}$ \\
\hline WN 2-4 & $2.6 \pm 0.3$ & $14 \mathrm{H}$ & & & 2 \\
WN 5 & $6.9 \pm 1.2$ & $12 \mathrm{H}$ & & & 2 \\
WN 6 & $10.4 \pm 1.6$ & $10 \mathrm{H}$ & & & 5 \\
WN 7 & $14.3 \pm 1.0$ & $12 \mathrm{H}$ & & & $10-20$ \\
WN 8-9 & $19.0 \pm 0.4$ & $14 \mathrm{H}$ & & & 20 \\
\hline WC 4 & $4.3 \pm 1.9$ & $2 \mathrm{~K}$ & & & 2 \\
WC 5 & $3.4 \pm 0.4$ & $7 \mathrm{~K}$ & 2.0 & $1 \mathrm{D}$ & 2 \\
WC 6 & $2.1 \pm 0.2$ & $7 \mathrm{~K}$ & 6.0 & $1 \mathrm{D}$ & 2 \\
WC 7 & $5.2 \pm 0.9$ & $7 \mathrm{~K}$ & 3.6 & $1 \mathrm{D}$ & 3 \\
WC 8 & $2.7 \pm 0.3$ & $2 \mathrm{~K}$ & $4.4 \pm 0.3$ & $2 \mathrm{D}$ & 4 \\
WC 9 & & & & & $10-20$ \\
\hline
\end{tabular}

$\mathrm{H}=$ Hamann \& Koesterke (1998a): WN-stars.

$\mathrm{K}=$ Koesterke \& Hamann (1995): WC-stars.

$\mathrm{D}=$ Dessart et al. (2000): WC-stars.

of values, we calculated models for both the upper and the lower limit of this range. For the hydrostatic core radius we adopt $R_{\mathrm{hc}}=R_{\text {evol }}$ if $R_{\mathrm{s}} / R_{\text {evol }}<2$ and $R_{\mathrm{hc}}=R_{\mathrm{s}} / 2$ if $R_{\mathrm{S}} / R_{\text {evol }}>2$. Note that reliable values of $R_{\text {evol }}$ are only available for WNE- and WC-stars.

\section{Optically thick wind models for typical WR-stars with fixed $\boldsymbol{R}_{\mathrm{s}}$}

We have applied the method for calculating optically thick radiatively driven wind models to the set of five WN-stars and three WC-stars of different spectral types. The parameters of these stars were discussed in Sect. 6 and are listed in Table 4. We have calculated optically thick wind models with input parameters: the mass and luminosity of the star, the chemical composition, mass-loss rate and the terminal velocity of the wind. The sonic radius was adopted according to the dependence between $R_{\mathrm{s}} / R_{\text {evol }}$ and spectral subclass (Table 5). The results of these models (variants A0 for constant $L(r)$ and B0 for $\chi \sim \rho / T^{3.5}$ ) are presented in Table 6 .

We see that the sonic points are at optical depths between about 3 and 33. The temperatures at the sonic point fall in two intervals, $40000<T_{\mathrm{s}}<80000 \mathrm{~K}$ and $140000<T_{\mathrm{s}}<190000 \mathrm{~K}$. We will show below that this is due to the dependence of the opacity in WR-stars on density and temperature: optically thick winds of WRstars can only exist if the temperature of the sonic point is near about $160000 \mathrm{~K}$ or near $50000 \mathrm{~K}$. The value of the velocity parameter $\beta$ is between about 3 and 6 for the WN-stars and between 4 and 7 for the WC-stars.

\subsection{Comparison with OPAL opacities}

The opacities in the vicinity of the sonic point for the models A0 and B0 can be compared with the OPAL opacities, which are the Rosseland mean opacities for non-expanding media (Iglesias \& Rogers 1993, 1996). In the wind models we need the flux-averaged opacities for the expanding media. In the case of optically thick winds the sonic points are located at large optical depths $\left(\tau^{\prime} \approx 20\right)$ with the velocities being around $30 \mathrm{~km} \mathrm{~s}^{-1}$ and at such conditions the Rosseland mean opacities are expected not to differ very much from the flux-averaged opacities. The models presented above showed that near the sonic point of WRwinds the temperatures are around $160000 \mathrm{~K}$ or $50000 \mathrm{~K}$. Therefore we only concentrate on the OPAL-opacities in the range of $4.5<\log (T)<5.5$.

In deriving the OPAL data we used the standard OPAL tables and the OPAL supportive codes for the interpolation from the tabulated data (hhtp://www-phys.1lnl.gov/Research/OPAL). We used the subroutine packet $\operatorname{OPACGN93}(Z, X, T 6, R)$ for WN-stars with metallicity $Z=0.02$ and with a hydrogen mass fraction $X$, with a temperature $T_{6}$ in millions of Kelvin, and with the OPAL parameter, $R=\rho / T_{6}^{3}$. For WC-stars we used the subroutine package $\operatorname{OPAC}(Z$, $X, X_{\mathrm{C}}, X_{\mathrm{O}}, T 6, R$ ) with $Z=0.02, X=0.0$ and the enhanced carbon mass fraction accounted for by the parameter $X_{\mathrm{C}}$ (the total mass fraction of $\mathrm{C}$ is the sum of the initial amount included in the metal mass fraction, $Z$, and $X_{\mathrm{C}}$ ), and the enhanced oxygen mass fraction accounted by the parameter $X_{\mathrm{O}}$ (the total mass fraction of $\mathrm{O}$ is the sum of the initial amount included in metal mass fraction, $Z$, and $X_{\mathrm{O}}$ ). The optically thick wind models of the WR-stars show that the parameter $R$ is in the range of $-7.0<\log (R)<-6.5$. The OPAL opacities for different values of $X$ and $Y$ with $Z=0.02$ are shown in the upper panels of Fig. 2 and those for C-enhanced and $\mathrm{H}$-free gas in the lower panels. Notice the strong bump in opacity in all models around $\log T \simeq 5.2$, which is mainly due to many bound-bound transitions of $\mathrm{Fe}$, and a very small bump around $\log T \simeq 4.6$ for some models, especially the C-rich and $\mathrm{H}$-free models.

We have compared the values of $\chi_{\mathrm{s}}$ and $(\mathrm{d} \chi / \mathrm{d} r)_{\mathrm{s}}$ of the optically thick wind models A0 and B0 of the WRstars (Table 6 ) with the OPAL values. We find that the values of $\chi_{\mathrm{s}}$ of the models are similar to the OPAL values. However, for all models there is a significant difference between the values of $(\mathrm{d} \chi / \mathrm{d} r)_{\mathrm{s}}$ of the models and those of the OPAL opacities. In all models this gradient is positive (this is a requirement of optically thick radiation driven winds, see Sect. 4.2), whereas the gradient of the OPAL opacities, for the temperatures and densities at the sonic point of the models, is in some cases even negative. We stress, however, that by changing somewhat the sonic radius $R_{\mathrm{s}}$ for models $\mathrm{A} 0$ and $\mathrm{B} 0$ it is possible to achieve exact agreement with the OPAL opacity $\chi_{\mathrm{s}}$ and the gradient $(\mathrm{d} \chi / \mathrm{d} r)_{\mathrm{s}}$ at the sonic point. This is because the OPAL opacity has a strong peak around $\log T \approx 5.2$ and a small peak near $\log T \approx 4.6$ (for $R \approx$ const.). Our models show that $R$ is about constant near the sonic point and so the plots of $\chi$ as function of $T$ in Fig. 2 for constant $R$ show approximately at which temperatures $(\mathrm{d} \chi / \mathrm{d} r)_{\mathrm{s}}$ can be positive. 
Table 6. Optically thick wind models for standard WR-stars: Variants A0 and B0.

\begin{tabular}{|c|c|c|c|c|c|c|c|c|c|c|c|c|c|c|}
\hline Star & Sp. & $\log L_{*}$ & $\log \dot{M}$ & $R_{\mathrm{s}}$ & $\begin{array}{l}\tau^{\prime}{ }_{\mathrm{s}} \\
\mathrm{A} 0\end{array}$ & $\begin{array}{l}\tau^{\prime}{ }_{\mathrm{s}} \\
\mathrm{B} 0\end{array}$ & $\begin{array}{r}T_{\mathrm{s}} \\
\mathrm{A} 0 \\
\end{array}$ & $\begin{array}{l}T_{\mathrm{s}} \\
\text { B0 }\end{array}$ & $\begin{array}{c}\beta \\
\mathrm{A} 0\end{array}$ & $\begin{array}{c}\beta \\
\text { B0 }\end{array}$ & $\begin{array}{c}\left(\frac{\mathrm{d} \chi}{\mathrm{d} r}\right)_{\mathrm{s}} \\
\mathrm{A} 0\end{array}$ & $\begin{array}{c}\left(\frac{\mathrm{d} \chi}{\mathrm{d} r}\right)_{\mathrm{s}} \\
\mathrm{B} 0\end{array}$ & $\begin{array}{l}\chi_{\mathrm{s}} \\
\mathrm{A} 0\end{array}$ & $\begin{array}{l}\chi_{\mathrm{s}} \\
\text { B0 }\end{array}$ \\
\hline WR2 & WN2 & 5.27 & -5.40 & 1.73 & 9.52 & 8.06 & 151600 & 145900 & 5.86 & 4.89 & 0.96 & 5.91 & 0.70 & 0.70 \\
\hline WR139 & WN5 & 5.21 & -5.04 & 1.65 & 22.6 & 19.2 & 183700 & 176800 & 5.92 & 5.03 & 1.45 & 10.4 & 0.76 & 0.76 \\
\hline WR136 & WN6 & 5.73 & -4.20 & 4.94 & 33.1 & 28.3 & 156900 & 150900 & 6.02 & 5.09 & 0.41 & 3.01 & 0.47 & 0.47 \\
\hline WR22 & WN7 & 6.08 & -4.38 & 16.22 & 9.57 & 8.10 & 79000 & 76000 & 5.26 & 4.34 & 0.13 & 0.76 & 0.60 & 0.60 \\
\hline WR22 & WN7 & 6.08 & -4.38 & 32.44 & 5.76 & 4.84 & 49700 & 47900 & 4.75 & 3.83 & 0.08 & 0.44 & 0.60 & 0.60 \\
\hline WR105 & WN9 & 5.81 & -4.55 & 26.3 & 3.67 & 3.05 & 42900 & 41300 & 3.77 & 2.93 & 0.12 & 0.62 & 0.44 & 0.44 \\
\hline WR111 & WC5 & 5.31 & -5.00 & 1.78 & 24.6 & 21.0 & 191200 & 184000 & 6.65 & 5.73 & 0.81 & 6.52 & 0.69 & 0.68 \\
\hline WR111 & WC5 & 5.31 & -5.00 & 2.67 & 18.5 & 15.7 & 145900 & 140400 & 6.53 & 5.56 & 0.61 & 4.14 & 0.68 & 0.68 \\
\hline WR42 & WC7 & 5.23 & -4.89 & 2.51 & 25.7 & 22.0 & 155500 & 149700 & 6.24 & 5.33 & 0.84 & 6.06 & 0.74 & 0.74 \\
\hline WR103 & WC9 & 5.20 & -4.62 & 8.18 & 20.6 & 17.5 & 80300 & 77300 & 5.89 & 4.89 & 0.48 & 2.74 & 0.76 & 0.76 \\
\hline WR103 & WC9 & 5.20 & -4.62 & 16.36 & 12.6 & 10.7 & 50600 & 48700 & 5.56 & 4.45 & 0.30 & 1.58 & 0.75 & 0.75 \\
\hline
\end{tabular}

(1) Variants A0 and B0 are for constant $L(r)$ and for $\chi \sim \rho / T^{3.5}$ respectively.

(2) $R_{\mathrm{s}}$ is in units of $R_{\odot}$ and $\dot{M}$ is in $M_{\odot} \mathrm{yr}^{-1}$.

(3) $(\mathrm{d} \chi / \mathrm{d} r)_{\mathrm{s}}$ is in units of $10^{-14} \mathrm{~cm} \mathrm{~g}^{-1}$ and $\chi_{\mathrm{s}}$ is in units of $\mathrm{cm}^{2} \mathrm{~g}^{-1}$.

\section{Optically thick wind models with OPAL opacities}

We compute a new set of models, called A1 and B1, using the OPAL opacities, with the abundances of the individual stars from Nugis \& Lamers (2000). In these models the sonic radius is varied (starting from the value of Table 5) until $(\mathrm{d} \chi / \mathrm{d} r)_{\mathrm{s}}$ becomes equal to that from the OPAL opacity tables. We were not trying to get exact agreement of the model values of $\chi_{\mathrm{s}}$ with the OPAL opacities, because the opacities in our models are the flux-mean opacities whereas the OPAL-opacities are the Rosseland-mean values. The flux-mean opacities are expected to be (slightly) higher than the Rosseland-mean values. In Variant A1 we assume that $L(r)$ is constant through the sonic point (similar to Variant A0) and in Variant B1 we assume that the opacity behaves like a power-law of the type $\chi=a \rho / T^{3.5}$ at the sonic point (similar to Variant B0).

The results are listed in Tables 7 and 8 for WN- and WC-stars. For WR105 (WN9) and WR103 (WC9) we present several models with different values of $R_{\mathrm{s}}$. These will be discussed below. The tables show the adopted input values for the stellar parameters $L_{*}$ and $\dot{M}$. The output values are: the radius, optical depth, temperature, opacity gradient and opacity, all at the sonic point. The OPAL-opacity gradient at the sonic point is per definition equal to that of the models. The opacity at the sonic point, that follows from the models, is compared with the OPAL-opacity at the same temperature and density. We see that the OPAL-opacities at the sonic point are typically a few tens of percent smaller than $\chi_{\mathrm{s}}$ (except for WR103). This could partly be due to the difference between the Rosseland-mean and the flux-mean opacities.

For the late type WR-stars WR105 and WR103 we present more than one model. This is because the sonic point radius $R_{\mathrm{S}}$ is not well known for the WN9 and WC9 stars. Starting with different values, we get different answers. If the resulting value of $R_{\mathrm{s}}$, that follows from the model calculations, differs more than a factor two from what we think is a reasonable value, the result is considered doubtful. The doubtful models in Tables 7 and 8 are given in brackets.

The sonic point temperatures of models A1 and B1 for each star are very similar. We see that the temperatures of the models at the sonic point fall into two regions, $156000<T_{\mathrm{s}}<162000 \mathrm{~K}$ and $37000<T_{\mathrm{s}}<71000 \mathrm{~K}$. It is a consequence of the fact that the opacity gradient $\mathrm{d} \chi / \mathrm{d} r$ has to be positive at the sonic point, to allow a transonic solution. The gradient is larger for models with a sonic point in the high temperature range, than for models with $T_{\mathrm{s}}$ in the low temperature range. However, the values of the opacity themselves are very similar in all cases. The models for stars with subtypes WN2 - WN6 and WC5 - WC7 all have high sonic point temperatures of $T_{\mathrm{s}} \approx 160000 \mathrm{~K}$. The star WR22 (WN7) has a solution both in the high and in the low temperature range. The star WR105 (WN9) has a sonic point in the low temperature range.

There is a problem with the models of WC9 star WR103. The low $T_{\mathrm{s}}$ models of this star require an opacity at the sonic point of $\chi_{\mathrm{s}} \approx 0.75 \mathrm{~cm}^{2} \mathrm{~g}^{-1}$, but the OPAL opacity at the sonic point is less than $0.30 \mathrm{~cm}^{2} \mathrm{~g}^{-1}$. On the other hand, the high temperature models for this star have about the right sonic point opacity, but the sonic point radius is much smaller than reasonable for this type. This discrepancy points either to a higher mass and luminosity than adopted for this star (this leads to a lower value of $\chi_{\mathrm{s}}$ ) or to a significant clumping at the sonic radius already, which would increase the Rosseland mean opacity.

\subsection{A bifurcation in the optically thick winds}

The optically thick wind models for WR-stars presented above clearly indicate the presence of two separate branches of solutions (bifurcation). These branches correspond to the intervals of sonic point temperature 
Table 7. Optically thick wind models for standard WR-stars: Variant A1 (constant $L(r))$.

\begin{tabular}{|c|c|c|c|c|c|c|c|c|c|c|}
\hline Star & Sp. & $\log L_{*}$ & $\log \dot{M}$ & $\begin{array}{l}R_{\mathrm{s}} \\
R_{\odot}\end{array}$ & $\tau_{\mathrm{s}}^{\prime}$ & $\begin{array}{r}T_{\mathrm{s}} \\
\mathrm{K} \\
\end{array}$ & $\begin{array}{c}\left(\frac{\mathrm{d} \chi}{\mathrm{d} r}\right)_{\mathrm{s}} \\
10^{-14} \mathrm{~cm} \mathrm{~g}^{-1}\end{array}$ & $\begin{array}{c}\chi_{\mathrm{s}} \\
\mathrm{cm}^{2} \mathrm{~g}^{-1} \\
\end{array}$ & $\begin{array}{c}\chi_{\mathrm{OPAL}} \\
\mathrm{cm}^{2} \mathrm{~g}^{-1}\end{array}$ & $\beta$ \\
\hline WR2 & WN2 & 5.27 & -5.40 & 1.64 & 9.87 & 156800 & 0.99 & 0.700 & 0.601 & 5.89 \\
\hline WR139 & WN5 & 5.21 & -5.04 & 2.06 & 19.3 & 158500 & 1.24 & 0.753 & 0.632 & 5.87 \\
\hline WR136 & WN6 & 5.73 & -4.20 & 4.82 & 33.7 & 159500 & 0.42 & 0.473 & 0.694 & 6.02 \\
\hline WR22 & WN7 & 6.08 & -4.38 & 22.7 & 7.49 & 63100 & 0.11 & 0.597 & 0.369 & 5.04 \\
\hline WR105 & WN9 & 5.81 & -4.55 & 14.5 & 5.81 & 63810 & 0.18 & 0.437 & 0.361 & 4.29 \\
\hline WR105 & WN9 & 5.81 & -4.55 & 32.7 & 3.09 & 37120 & 0.10 & 0.435 & 0.371 & 3.54 \\
\hline WR111 & WC5 & 5.31 & -5.00 & 2.39 & 20.0 & 157300 & 0.66 & 0.681 & 0.539 & 6.57 \\
\hline WR42 & $\mathrm{WC} 7$ & 5.23 & -4.89 & 2.44 & 26.2 & 158300 & 0.86 & 0.737 & 0.568 & 6.24 \\
\hline WR103 & WC9 & 5.20 & -4.62 & 9.90 & 18.0 & 70750 & 0.42 & 0.755 & 0.261 & 5.82 \\
\hline (WR103 & WC9 & 5.20 & -4.62 & 2.93 & 42.3 & 159300 & 0.98 & 0.774 & 0.602 & $6.03)$ \\
\hline (WR103 & WC9 & 5.20 & -4.62 & 23.4 & 9.79 & 39900 & 0.23 & 0.750 & 0.296 & $5.28)$ \\
\hline
\end{tabular}

Table 8. Optically thick wind models for standard WR-stars: Variant B1 $\left(\chi \sim \rho / T^{3.5}\right)$.

\begin{tabular}{lllllllcrcc}
\hline \hline Star & Sp. & $\log L_{*}$ & $\log \dot{M}$ & $\begin{array}{c}R_{\mathrm{s}} \\
R_{\odot}\end{array}$ & $\tau_{\mathrm{s}}^{\prime}$ & $\begin{array}{r}T_{\mathrm{s}} \\
\mathrm{K}\end{array}$ & $\begin{array}{c}\left(\frac{\mathrm{d} \chi}{\mathrm{d} r}\right)_{\mathrm{s}} \\
10^{-14} \mathrm{~cm} \mathrm{~g}^{-1}\end{array}$ & $\begin{array}{c}\chi_{\mathrm{s}} \\
\mathrm{cm}^{2} \mathrm{~g}^{-1}\end{array}$ & $\begin{array}{c}\chi_{\mathrm{OPAL}} \\
\mathrm{cm}^{2} \mathrm{~g}^{-1}\end{array}$ & $\beta$ \\
\hline WR2 & WN2 & 5.27 & -5.40 & 1.53 & 8.82 & 158500 & 6.64 & 0.700 & 0.609 & 4.97 \\
WR139 & WN5 & 5.21 & -5.04 & 1.91 & 17.3 & 160100 & 8.91 & 0.753 & 0.650 & 4.98 \\
WR136 & WN6 & 5.73 & -4.20 & 4.47 & 30.4 & 161300 & 3.37 & 0.473 & 0.705 & 5.12 \\
WR22 & WN7 & 6.08 & -4.38 & 5.40 & 17.9 & 158200 & 2.31 & 0.608 & 0.756 & $4.83)$ \\
WR105 & WN9 & 5.81 & -4.55 & 28.9 & 2.82 & 38730 & 0.58 & 0.435 & 0.374 & 2.84 \\
\hline WR111 & WC5 & 5.31 & -5.00 & 2.22 & 18.0 & 158900 & 5.06 & 0.681 & 0.548 & 5.65 \\
WR42 & WC7 & 5.23 & -4.89 & 2.28 & 23.5 & 159700 & 6.76 & 0.737 & 0.578 & 5.36 \\
WR103 & WC9 & 5.20 & -4.62 & 9.82 & 15.4 & 68450 & 2.36 & 0.755 & 0.266 & 4.79 \\
(WR103 & WC9 & 5.20 & -4.62 & 2.72 & 38.1 & 161300 & 8.38 & 0.773 & 0.613 & $5.17)$ \\
(WR103 & WC9 & 5.20 & -4.62 & 20.8 & 9.03 & 41510 & 1.32 & 0.750 & 0.300 & $4.26)$ \\
\hline
\end{tabular}

where it is possible to achieve positive opacity gradients $\left((\mathrm{d} \chi / \mathrm{d} r)_{\mathrm{s}}>0\right)$ : the high-temperature regime with $T \simeq 156000 \mathrm{~K}$ and the low-temperature regime with $37000 \leq T \leq 71000 \mathrm{~K}$. The high-temperature range is connected with the well-known iron opacity peak around $\log T \approx 5.2$ and the low-temperature range is connected with the weak opacity enhancement due to lower ions of iron and other metals in the range $37000 \leq T \leq 71000 \mathrm{~K}$. As can be seen from the OPAL-opacity tables, these ranges are clearly separated because $\mathrm{d} \chi / \mathrm{d} r<0$ for $71000 \leq T \leq$ $156000 \mathrm{~K}$. This means that in the case of optically thick wind models with negligible contribution from the line driving force due to expansion, it is not possible to have a smooth evolution from the regime of mass loss with sonic point temperatures in the low-temperature range to the regime with sonic point temperatures with $T \geq 156000 \mathrm{~K}$.

\section{Discussion}

The optically thick radiation driven wind models for WRstars derived in this paper differ from the "standard" models of WR-winds (Hamann \& Koesterke 1998a; Koesterke \& Hamann 1995; Dessart et al. 2000) in several ways: the velocity-law parameter $\beta \approx 4$ to 6 of our models is considerably higher than the adopted $\beta=1$ of the "standard" models and the sonic point radius of the optically thick models is smaller than the inner radius of the "standard" models for early type WR-stars.

The low value of $\beta$ is approximately correct for the winds of O-stars (e.g. Haser et al. 1995; Puls et al. 1996; Herrero et al. 2000), but for the winds of WR-stars $\beta$ might be significantly higher. This is because in O-star winds the radiative acceleration is mainly due to spectral lines, and hence the radiation force is sensitive to the Doppler shifts produced by the velocity gradient. This results in a fast acceleration of the wind and a small value of $\beta \simeq 0.7$ to 1 (e.g. Lamers \& Cassinelli 1999, p. 240). However, the optically thick winds of WR-stars are largely driven by opacity sources which are less sensitive to Doppler shifts and hence we can expect a slower acceleration and higher values of $\beta$. This is supported by the analysis of the spectroscopic data of WR-stars by Lépine \& Moffat (1999) (see also Moffat \& Lépine 2000). They studied the variations of subpeaks in the line profiles of WR-stars and, assuming that these subpeaks are due to propagating wind inhomogeneities, they find that $\beta \simeq 5$ to 10 . (A similar analysis of the line profile variations of the star $\zeta$ Pup (O4If) by Eversberg et al. (1998) gives $\beta \approx 1-1.2$, in very good agreement with $\beta \simeq 1.15$ derived from modeling of the $H_{\alpha}$-profile (Puls et al. 1996). This supports the assumption that the study of the kinematics of the subpeaks provides a 

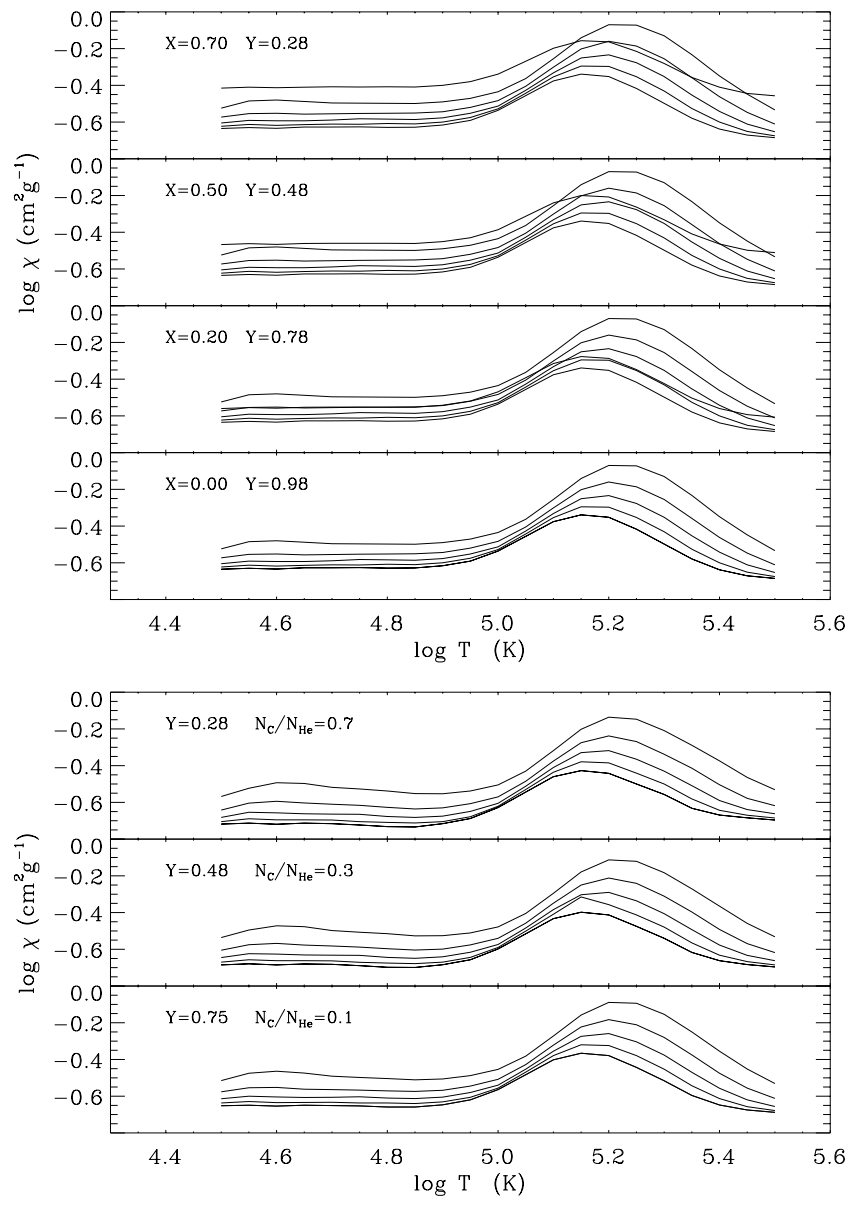

Fig. 2. The OPAL opacity for different abundances as a function of temperature and $R \equiv \rho /\left(T_{6}^{3}\right)$. The plotted data are for $\log R=-8.0,-7.5,-7.0,-6.5$ and -6.0 . The higher the value of $R$ the higher the values of $\chi$ at $\log T=4.5$ and 5.5. The top figure is for models with $Z=0.02$ but different $\mathrm{H}$ and $\mathrm{He}$ abundances. The lower figure is for $\mathrm{H}$-free gas with $Z=0.02$ and different abundances of He and C. Notice the strong bump near $\log (T) \approx 5.2$ and the small bump near $\log (T) \approx 4.6$. The sonic points of optically thick winds can only occur in the temperature regions where $\mathrm{d} \chi / \mathrm{d} T>0$.

reasonably good estimate of the value of $\beta$. Further support for the high value of $\beta$ for WR-stars comes from the study of the hydrodynamical modeling of the wind of WN4b star HD50896 by Schmutz (1997), who derived $\beta \simeq 8$. Hillier \& Miller (1999) concluded that the atmospheric models with values of $\beta$ higher than 1 can not be excluded, but that it is very difficult to constrain the velocity law in the standard spectroscopic modeling studies, particularly for WC-stars with severe blending of lines.

We are aware of the fact that optically thick (in continuum) wind models are more sensitive to the velocity law in the inner part of the wind than in the outer wind and therefore the actual value of $\beta$ may be smaller in the part of the wind where the observed spectral lines are effectively formed. We assumed in our paper that $\beta$ is the same in the whole wind. However this was not a crucial assumption. It only allowed us to give a rough estimate of the "mean" value of $\beta$ that is needed for a sufficiently large optical depth at the sonic point.

Support for our conclusion that the sonic radius $R_{\mathrm{s}}$ is smaller than the inner radius ("core" radius) of the "standard" models comes from the analysis of eclipsing and spectroscopic binaries. Cherepashchuk (1991, 2000) found from the modeling of light curves of eclipsing binary system V444 Cyg (HD 193576, WR139) that the optical photospheric radius where $\tau_{\mathrm{V}} \approx 1$ is at $R_{\mathrm{ph}} \simeq 3 R_{\odot}$ for the WN5-component. Hamann \& Schwarz (1992) derived by the standard atmospheric modeling that $R_{\tau 20} \approx 6 R_{\odot}$ for this WN 5 star, which is twice as large. For the another eclipsing binary system CQ Cep (HD 214419, WR155) it is estimated from the analysis of the eclipses and the orbital motion that $R_{\mathrm{ph}} \leq 10 R_{\odot}$ for the WN6component (Cherepashchuk 1991; Marchenko et al. 1995; Moffat \& Marchenko 1996). On the other hand Hamann \& Koesterke (1998a) derived from the standard atmospheric modeling study that $R_{\tau 20} \approx 25 R_{\odot}$ for this WN6component. The radius $R_{\tau 20}$ of the inner boundary of the atmospheric models at $\tau \simeq 20$ should be smaller than the optical radius. However, we see that for both binary systems the derived values of $R_{\tau 20}$ are about twice larger than the empirically derived photospheric radii. We can conclude that the "stardard" atmospheric models of WRstars overestimate the core radii of early type WR-stars by about a factor 2 to 3 . Therefore, the fact that our models require a smaller sonic radius than $R_{\tau 20}$ of the "standard" models and a higher value of $\beta$ is very reasonable.

\section{Summary and conclusions}

The existing problems of the optically thin radiation driven wind theory in explaining the high momentum and the high mass-loss rates of WR-stars, has prompted our investigation of the possibility that the high mass-loss rates of WR-stars may be due to optically thick radiation driven winds.

Adopting the stellar parameters of the WR-stars and their observed high mass-loss rates and terminal wind velocities, we investigated the conditions at the sonic point of the wind that are needed to explain the high mass-loss rates. We reached the following conclusions:

1. The mass-loss rates of optically thick radiation driven winds is approximately

$\dot{M} \simeq c_{1} a_{1}^{1 / 2} R_{\mathrm{s}}^{3} T_{\mathrm{s}}^{4.5} / G M$

with $c_{1}=64 \pi \sigma /(3 c)$ and $a_{1}=k(\gamma+1) /\left(\mu m_{\mathrm{H}}\right)$, where $R_{\mathrm{S}}$ and $T_{\mathrm{s}}$ are the radius and temperature at the sonic point $\left(v_{\mathrm{s}}=c_{\mathrm{S}}\right), \gamma$ is the mean number of free electrons per ion and $\mu$ is the mean atomic weight of the atoms.

2. Optically thick radiation driven wind models require that the opacity gradient at the sonic point is positive, i.e. $(\mathrm{d} \chi / \mathrm{d} r)_{\mathrm{s}}>0$. The OPAL-opacities for WR-stars show that this occurs in two temperature regimes: a hot regime with $155000<T_{\mathrm{s}}<165000 \mathrm{~K}$, and a cool regime with $37000<T_{\mathrm{s}}<71000 \mathrm{~K}$. The sonic points of early-type WR-stars (WN2-WN6 and WC5-WC7) 
are in the hot regime and those of late-type WR-stars are in the cool regime.

3. The high sonic point temperatures imply a rather large effective optical depth at the sonic point of $\tau_{\mathrm{s}}^{\prime} \simeq 3$ to 10 for the low temperature regime and about 10 to 30 for the high temperature regime. Such high effective optical depths for the sonic points of WR-stars can only be achieved if the velocity law in the supersonic region is rather "soft", with $\beta \approx 4$ to 6 . There is indeed observational evidence that winds of WR-stars have such soft velocity laws.

4. The values of the opacities at the sonic points of our models that are required to explain the observed massloss rates of the WR-stars are close to the OPALopacities for the abundances of these WR-stars.

5. The sonic radii $R_{\mathrm{S}}$ of our models are smaller than the so-called "core-radii" where $\tau \simeq 20$ of the "standard" models for early type WR-stars by about a factor two. Studies of WR-stars in eclipsing binary systems support the smaller radii of our models.

6. Taking all these facts together, we find that the high mass-loss rates of WR-stars can be explained by optically thick radiation driven wind models.

We point out that we did not solve the structure of the whole wind. In fact, we only considered the conditions at the sonic point that are needed to start the wind with a high mass-loss rate. In particular, we did not consider the acceleration of the wind in the supersonic region. Therefore, if our assumption that the high mass-loss rate of WR-stars is due to radiation pressure in the optically thick transonic region is correct, we still have solved only half of the problem. The continuous acceleration of the outflowing gas up to the observed high terminal velocities still remains to be explained. The smaller radii and the more slowly increasing velocity laws that we derived, compared to the usually assumed values, may help in this respect, because a smaller radius implies a larger radiative flux (for the same luminosity) and a softer velocity law requires a smaller acceleration of the wind.

In a subsequent paper we will discuss the consequences of the possible occurrence of three types of radiation driven wind models (optically thin line-driven winds, optically thick radiation driven winds with $T_{\mathrm{s}} \approx 160000 \mathrm{~K}$, and optically thick radiation driven winds with $T_{\mathrm{s}} \approx$ $50000 \mathrm{~K}$ ) during the evolution of massive stars.

Acknowledgements. This work was supported by the Estonian Science Foundation grants No. 3166 and 5003, by a visitorgrant from NOVA, The Netherlands Research School for Astronomy and travel grants from the Leidsch KerkhovenBosscha Foundation. We thank the referee, W.-R. Hamann, for constructive comments on an earlier version of the paper.

\section{References}

Cassinelli, J. P. 1991, Wolf-Rayet Stars and Interrelations with Other Massive Stars in Galaxies, Proc. IAU Symp. 143, ed. K. A. van der Hucht, \& B. Hidayat (Kluwer), 289
Cassinelli, J. P., \& Castor, J. I. 1973, ApJ, 179, 189

Castor, J. I., Abbott, D. C., \& Klein, R. I. 1975, ApJ, 195, 157

Cherepashchuk, A. M. 1991, Wolf-Rayet Stars and Interrelations with Other Massive Stars in Galaxies, Proc. IAU Symp. 143, ed. K. A. van der Hucht, \& B. Hidayat (Kluwer), 187

Cherepashchuk, A. M. 2000, in ASP Conf. Ser. 204, Thermal and Ionization Aspects of Flows From Hot Stars: Observations and Theory, ed. H. J. G. L. M. Lamers, \& A. Sapar, 249

Dessart, L., Crowther, P. A., Hillier, D. J., et al. 2000, MNRAS, 315,407

Eversberg, T., Lépine, S., \& Moffat, A. F. J. 1998, ApJ, 494, 799

Gayley, K. G., Owocki, S. P., \& Cranmer, S. R. 1995, ApJ, 442,296

Hamann, W.-R., \& Koesterke, L. 1998a, A\&A, 333, 251

Hamann, W.-R., \& Koesterke, L. 1998b, A\&A, 335, 1003

Hamann, W.-R., \& Schwarz, E. 1992, A\&A, 261, 523

Hamann, W.-R., Koesterke, L., \& Gräfener, G. 2000, in Thermal and Ionization aspects of Flows from Hot Stars: Observations and Theory, ed. H. J. G. L. M. Lamers, \& A. Sapar, ASP Conf. Ser., 204, 197

Haser, S., Lennon, D. J., \& Kudritzki, R.-P. 1995, A\&A, 295, 136

Heger, A., \& Langer, N. 1996, A\&A, 315, 421

Herrero, A., Puls, J., \& Villamariz, M. R. 2000, A\&A, 354, 193

Hillier, D. J., \& Miller, D. L. 1999, ApJ, 519, 354

Iglesias, C. A., \& Rogers, F. J. 1993, ApJ, 412, 752

Iglesias, C. A., \& Rogers, F. J. 1996, ApJ, 464, 943

Kato, M., \& Iben, I. Jr. 1992, ApJ, 394, 305

Koesterke, L., \& Hamann, W.-R. 1995, A\&A, 299, 503

Lamers, H. J. G. L. M., \& Cassinelli, J. P. 1999, Introduction to Stellar Winds (Cambridge Univ. Press)

Langer, N. 1989, A\&A, 210, 93

Lépine, S., \& Moffat, A. F. J. 1999, ApJ, 514, 909

Lucy, L. B. 1971, ApJ, 163, 95

Lucy, L. B., \& Abbott, D. C. 1993, ApJ, 405, 738

Maeder, A. 1991, A\&A, 242, 93

Maeder, A., \& Meynet, G. 1987, A\&A, 182, 243

Marchenko, S. V., Moffat, A. F. J., Eenens, P. R. J., Hill, G. M., \& Grandchamps, A. 1995, ApJ, 450, 811

Mihalas, D. 1978, Stellar Atmospheres (W.H. Freeman and Company, San Francisco)

Moffat, A. F. J., \& Marchenko, S. V. 1996, A\&A, 305, L29

Moffat, A. F. J., \& Lépine, S. 2000, in ASP Conf. Ser., 204, Thermal and Ionization Aspects of Flows From Hot Stars: Observations and Theory, ed. H. J. G. L. M. Lamers, \& A. Sapar, 15

Nugis, T., Crowther, P. A., \& Willis, A. J. 1998, A\&A, 333, 956

Nugis, T., \& Lamers, H. J. G. L. M. 2000, A\&A, 360, 227

Owocki, S. P., \& Gayley, K. G. 1999, Wolf-Rayet Phenomena in Massive Stars and Starburst Galaxies, Proc. IAU Symp. 193, ed. K. A. van der Hucht, G. Koenigsberger, \& P. R. J. Eenens, ASP, 157

Pistinner, S., \& Eichler, D. 1995, ApJ, 454, 404

Puls, J., Kudritzki, R.-P., Herrero, A., et al. 1996, A\&A, 305, 171

Quinn, T., \& Paczyński, B., ApJ, 289, 634

Schaerer, D., \& Maeder, A. 1992, A\&A, 263, 129

Schmutz, W. 1997, A\&A, 321, 268 